BULLETIN (New Series) OF THE AMERICAN MATHEMATICAL SOCIETY

Volume 34, Number 3, July 1997, Pages 251-292

S 0273-0979(97)00727-1

\title{
LECTURES ON AFFINE HECKE ALGEBRAS AND MACDONALD'S CONJECTURES
}

\author{
ALEXANDER A. KIRILLOV, JR.
}

\begin{abstract}
This paper gives a review of Cherednik's results on the representation-theoretic approach to Macdonald polynomials and related special functions. Macdonald polynomials are a remarkable 2-parameter family of polynomials which can be associated to every root system. As special cases, they include the Schur functions, the $q$-Jacobi polynomials, and certain spherical functions on real and $p$-adic symmetric spaces. They have a number of elegant combinatorial properties, which, however, are extremely difficult to prove. In this paper we show that a natural setup for studying these polynomials is provided by the representation theory of Hecke algebras and show how this can be used to prove some of the combinatorial identities for Macdonald polynomials.
\end{abstract}

\section{INTRODUCTION}

This paper is based on a series of lectures delivered by the author at Harvard University in the Fall of 1994. The aim of these lectures was to give a self-contained exposition of the recent result of Cherednik ([C6]), who proved Macdonald's inner product identities (see [M2]) for arbitrary root systems, using the algebraic structure he called "double affine Hecke algebra". These identities, conjectured by Macdonald, give a very elegant formula for the norms of Macdonald polynomials a remarkable two-parameter family of orthogonal polynomials associated with root systems. Macdonald polynomials have been the object of intensive study since their introduction in 1988, and a lot of special cases of the inner product identities have been proved; however, no uniform proof which would work for all root systems was known. The major discovery of Cherednik was that Macdonald polynomials naturally appear in the representation theory of affine Hecke algebras, which provided him with the tools for proving the inner product identities.

These lectures were intended for non-specialists; for this reason all the necessary definitions (including that of Macdonald polynomials and Hecke algebras) and motivations are included in the course. Some technical details of the proofs are omitted; an experienced reader can always fill in the gaps. However, it is assumed that the reader is familiar with finite-dimensional and affine root systems and Weyl groups. A short introduction to these notions can be found in [Hu1, Chapter III]; for more detailed expositions we refer the reader to $[\mathrm{B}],[\mathrm{Hu} 2]$. See also a recent survey of Koornwinder [Ko1] for a discussion of the relation between the geometry of root systems and the theory of orthogonal polynomials.

Received by the editors January 19, 1995, and, in revised form, April 3, 1997.

1991 Mathematics Subject Classification. Primary 05E35; Secondary 33D80.

The author was supported by Alfred P. Sloan dissertation fellowship. 
These lectures are of an expository nature and do not contain any new results. I include the references to the original papers in each lecture. Also, I must stress that these lectures were written under the strong influence of the (unpublished) courses of Ivan Cherednik (Yale, Fall 1991) and Howard Garland (Yale, Spring 1993) and a series of talks by Ian Macdonald (Yale, October 1993, and Leiden University, May 1994). A large part of my lectures (most of Lectures 2-4,6,7) follow Macdonald's exposition, so all credits for these lectures belong to him; of course, I am completely responsible for any errors that could be found in these notes.

\section{ACKNOWLEDGMENTS}

I am deeply grateful to Ivan Cherednik for numerous discussions, in which he explained to me many parts of this theory. Without these discussions, my lectures would never have come to a happy end.

I would like to thank the mathematics department of Harvard University for its hospitality during my work on these lectures and Professors Tom Koornwinder, Masatoshi Noumi, and Sergei Fomin for their valuable remarks on the preliminary version of these notes. Finally, I want to thank Joshua Scott for correcting my English.

Note added in proof. After this paper was submitted for publication, the author received a copy of a review by Macdonald [M7], which serves the same purpose as our paper. However, our exposition is somewhat different from Macdonald's, so we suggest that the reader look at both papers to get a better perspective.

\section{LECTURE 0: History AND MOTIVATIONS}

These lectures are devoted to the study of a remarkable family of symmetric polynomials - Macdonald polynomials, and in particular, to the proof of Macdonald's inner product identities. We give here a brief introduction to these notions and try to explain their importance. Formal definitions will be given later on; therefore, readers who are not interested in motivations can skip this lecture.

Let us start with the algebra $\mathbb{C}[\mathbf{x}]=\mathbb{C}\left[x_{1}, \ldots, x_{n}\right]$. For brevity, for a multiindex $\lambda=\left(\lambda_{1}, \ldots, \lambda_{n}\right) \in \mathbb{Z}_{+}^{n}$ we denote $\mathbf{x}^{\lambda}=x_{1}^{\lambda_{1}} \ldots x_{n}^{\lambda_{n}}$. The symmetric group $S_{n}$ naturally acts on $\mathbb{C}[\mathbf{x}]$, and therefore we can define the subspace of symmetric polynomials $\Lambda_{n} \subset \mathbb{C}[\mathbf{x}]$. Note that we are treating $\Lambda_{n}$ as a vector space, not as an algebra.

The space $\Lambda_{n}$ plays an important role in combinatorics and has been thoroughly studied. We refer the reader to the excellent monograph by Macdonald [M6] for detailed information. We will be interested in only a small part of the theory: we will construct certain bases in $\Lambda$.

The simplest basis is given by the monomial functions $m_{\lambda}$, which are defined as follows. Denote by $\mathcal{P}^{n}$ the set of partitions of length $n$, i.e. the set of $\lambda=$ $\left(\lambda_{1}, \ldots, \lambda_{n}\right) \in \mathbb{Z}_{+}^{n}$ satisfying the condition

$$
\lambda_{1} \geq \lambda_{2} \cdots \geq \lambda_{n} \geq 0 .
$$

We define the monomial functions $m_{\lambda}$ by

$$
m_{\lambda}=\sum_{\mu \in S_{n}(\lambda)} \mathbf{x}^{\mu}, \quad \lambda \in \mathcal{P}^{n}
$$


where $S_{n}(\lambda)$ is the orbit of $\lambda$ under the action of the symmetric group, i.e. the set of all $\mu \in \mathbb{Z}_{+}^{n}$ that can be obtained from $\lambda$ by a permutation of components. For example, if $\lambda=(r, 0, \ldots, 0)$, then $m_{\lambda}=\sum_{i=1}^{n} x_{i}^{r}$. The elements $m_{\lambda}$ form a basis in $\Lambda_{n}$, which follows from the obvious fact that every $S_{n}$-orbit in $\mathbb{Z}_{+}^{n}$ contains a unique partition.

Another important basis in the space of symmetric polynomials is given by the Schur functions, which are defined by

$$
s_{\lambda}=\frac{\sum_{w \in S_{n}} \operatorname{sgn}(w) \mathbf{x}^{w(\lambda+\rho)}}{\prod_{i<j}\left(x_{i}-x_{j}\right)}, \quad \lambda \in \mathcal{P}^{n}
$$

where $\operatorname{sgn}(w)$ is the sign of $w$, and $\rho=(n-1, n-2, \ldots, 0) \in \mathbb{Z}_{+}^{n}$. Even though formula (0.3) is written as a fraction, Schur functions are in fact polynomials, since every antisymmetric polynomial is divisible by $\prod_{i<j}\left(x_{i}-x_{j}\right)$. It is easy to show that the matrix, expressing $s_{\lambda}$ in terms of $m_{\lambda}$, is triangular:

$$
s_{\lambda}=m_{\lambda}+\text { linear combination of } m_{\mu} \text { with } \mu<\lambda,
$$

where $<$ is the lexicographic order on partitions: $\mu<\lambda$ if $\sum \lambda_{i}=\sum \mu_{i}$ and $\mu_{1}=\lambda_{1}, \ldots, \mu_{k-1}=\lambda_{k-1}, \mu_{k}<\lambda_{k}$ for some $k$.

The Schur functions have a number of remarkable properties (see [M6]); for example, they naturally appear as the characters of irreducible representations of the group $G L_{n}(\mathbb{C})$.

Both the monomial functions and the Schur functions can also be defined by certain orthogonality properties. Let us consider elements of the space $\mathbb{C}[\mathbf{x}]$ as complex-valued functions on the $n$-dimensional real vector space $\mathbb{R}^{n}=\left\{\left(t_{1}, \ldots, t_{n}\right)\right\}$. This can be done by assigning to the variable $x_{k}$ the function $x_{k}\left(t_{1}, \ldots, t_{n}\right)=e^{2 \pi \mathrm{i} t_{k}}$ and then extending it by multiplicativity and linearity. Note that $\overline{x_{k}}=x_{k}^{-1}$. These functions are well-defined on the torus $T=\mathbb{R}^{n} / \mathbb{Z}^{n}$, and

$$
\int_{T} x^{\lambda} d \mathbf{t}=\left\{\begin{array}{lc}
1, & \lambda=0 \\
0 & \text { otherwise }
\end{array}\right.
$$

The monomial basis can be uniquely characterized by the following two conditions:

(i) The triangularity condition:

$$
m_{\lambda}=\mathbf{x}^{\lambda}+\text { lower terms, }
$$

where "lower terms" means a linear combination of $\mathbf{x}^{\mu}$ with $\mu<\lambda$.

(ii) The orthogonality condition:

$$
\int_{T} m_{\lambda} \overline{m_{\mu}} d \mathbf{t}=0 \quad \text { for } \lambda \neq \mu \text {. }
$$

The Schur functions $s_{\lambda}$ can be defined in a similar manner. Using the explicit formula (0.3), it is not hard to show that $s_{\lambda}$ are uniquely defined by the triangularity condition similar to (0.4) and the following orthogonality condition:

$$
\int_{T} \prod_{i<j}\left|x_{i}-x_{j}\right|^{2} s_{\lambda} \overline{s_{\mu}} d \mathbf{t}=0 \quad \text { for } \lambda \neq \mu .
$$

Having noticed this, it is natural to ask what happens if we consider a more general orthogonality condition. Let us fix $k \in \mathbb{Z}_{+}$and define $J_{\lambda}^{k} \in \Lambda_{n}$ by the same 
triangularity condition (0.4) together with the following orthogonality condition:

$$
\int_{T} \prod_{i<j}\left|x_{i}-x_{j}\right|^{2 k} J_{\lambda}^{k} \overline{J_{\mu}^{k}} d \mathbf{t}=0 \quad \text { for } \lambda \neq \mu .
$$

Note that since $\overline{x_{i}-x_{j}}=x_{i}^{-1}-x_{j}^{-1}$, the kernel $\prod\left|x_{i}-x_{j}\right|^{2 k}$ can be rewritten as follows:

$$
\prod_{i<j}\left|x_{i}-x_{j}\right|^{2 k}=\prod_{i \neq j}\left(1-\frac{x_{i}}{x_{j}}\right)^{k} .
$$

As before, it can be shown that these conditions uniquely define the polynomials $J_{\lambda}^{k}$. They are called the Jack polynomials (see [J], [St]). For $k=0,1$ the Jack polynomials coincide with the monomial functions and with the Schur functions, respectively. For $k=1 / 2$ these polynomials can be interpreted as zonal spherical functions on certain symmetric spaces (see $[\mathrm{St}]$ ).

Of course, we could have replaced the kernel $\prod\left|x_{i}-x_{j}\right|^{2 k}$ by almost any symmetric function and used it to define a family of orthogonal polynomials. However, the Jack polynomials have a number of interesting properties which single them out. Here is one of them: these polynomials (after some simple renormalization) are the eigenfunctions of the following second order differential operator:

$$
L_{2}=\sum \frac{\partial^{2}}{\partial t_{i}^{2}}+k(k-1) \sum_{i \neq j} \frac{1}{2 \sin ^{2}\left(\frac{t_{i}-t_{j}}{2}\right)} .
$$

In the physical language, this is the Schrödinger operator for a quantum-mechanical system of $n$ points on a circle with the potential of pairwise interaction proportional to $1 / r^{2}$, where $r$ is the distance between the points. This system was studied by Sutherland([Su] $)$ and Calogero, Ragnisco and Marchioro ([CRM]), who proved that it is completely integrable: there are $n$ algebraically independent symmetric differential operators which commute with each other and with $L_{2}$. These differential operators play the same role the first integrals, or conservation laws, play in classical mechanics.

It should be noted that completely integrable systems occupy a very special place in quantum mechanics. In a certain sense, they are the only systems allowing complete analysis. However, very few examples of completely integrable systems are known.

Another striking property of Jack polynomials is related with the calculation of the norms

$$
\left\|J_{\lambda}^{k}\right\|^{2}=\frac{1}{n !} \int_{T} \prod\left|x_{i}-x_{j}\right|^{2 k} J_{\lambda}^{k}(t) \overline{J_{\lambda}^{k}(t)} d t .
$$

In the cases $k=0,1$, which correspond to the monomial basis $m_{\lambda}$ and the Schur functions $s_{\lambda}$ respectively, this is easy to do. The answer is $\left|m_{\lambda}\right|^{2}=1 /\left|W_{\lambda}\right|,\left|s_{\lambda}\right|^{2}=$ 1 , where $W_{\lambda}$ is the stabilizer of $\lambda$ in the symmetric group $S_{n}$.

In general, however, computing these norms is a very difficult combinatorial problem. One might expect to get a very complicated formula. However, by some miracle, the answer is given by a very simple product (see [M6]):

$$
\left\|J_{\lambda}^{k}\right\|^{2}=\prod_{i<j} \prod_{m=0}^{k-1} \frac{\lambda_{i}-\lambda_{j}+k(j-i)+m}{\lambda_{i}-\lambda_{j}+k(j-i)-m} .
$$


This formula is non-trivial even for $\lambda=0$. In this case, $J_{0}^{k}=1$ and therefore the calculation of the norm reduces to the calculation of the constant term of $\prod_{i \neq j}\left(1-\frac{x_{i}}{x_{j}}\right)^{k}$. It is an instructive exercise to check that for $\lambda=0$, formula $(0.9)$ can be rewritten as follows:

$$
\text { Constant term }\left(\prod_{i \neq j}\left(1-\frac{x_{i}}{x_{j}}\right)^{k}\right)=\frac{(n k) !}{(k !)^{n}} .
$$

This is a special case of Dyson formula, conjectured by Dyson ([Dy]) in 1962 and proved in $[\mathrm{Gu}],[\mathrm{W}]$ (see also [Go]).

Both the relation with integrable systems and the combinatorial formulas like $(0.10)$ show that the Jack polynomials are indeed a very special family of symmetric polynomials. But it turns out that it is just the beginning. The Jack polynomials can be generalized in at least two ways. First, one can replace the symmetric group $S_{n}$ by an arbitrary Weyl group (or even more general Coxeter group), acting on the appropriate analogue of the space of polynomials. The definition of the Jack polynomials can be generalized to this case, which was done by Heckman and Opdam in $[\mathrm{HO}]$; they called these new polynomials "Jacobi polynomials associated with root systems".

Second, one can introduce a $q$-analogue of these polynomials by replacing the product $\left(x_{i}-x_{j}\right)^{k}$ by $\prod_{i=0}^{k-1}\left(x_{i}-q^{2 i} x_{j}\right)$, and similarly for other root systems. This gives a family of polynomials depending on $q$. The obtained polynomials are called the Macdonald polynomials (see [M1], [M2]). As special cases, these polynomials not only include the Jack polynomials, but also Hall polynomials and certain spherical functions on $p$-adic groups, which shows that it is a truly remarkable family of polynomials.

A question that naturally arises is, do the Macdonald polynomials have the same nice properties as the Jack polynomials. For example, can they be described as eigenfunctions of a commuting family of differential (or difference) operators? Are there analogues of the constant term identity (0.10), and, more generally, of the inner product identity (0.9)? Can one describe what are the denominators of the coefficients of these polynomials?

These and many other questions were studied by many mathematicians, starting with Macdonald himself, who proved the inner product identity for the group $S_{n}$ and conjectured an analogue of formula (0.9) for arbitrary Weyl group (see Theorem 2.4 below). Many other special cases were proved case-by-case (see references in Lecture 2). However, all the attempts to find a uniform proof within the framework of combinatorics or the theory of special functions failed.

The breakthrough was achieved by Cherednik, who used representation-theoretic methods. Roughly speaking, the main idea of these methods can be formulated as follows: if we show that the Macdonald polynomials naturally appear in the representation theory of some algebraic structure, such as a group or an algebra, then we can translate known properties of this structure into statements about Macdonald polynomials. For example, the Jack polynomials with $k=\frac{1}{2}$, as was mentioned above, appear as zonal spherical functions on certain symmetric spaces for $S L_{n}$, and the differential operator $L_{2}$, given by $(0.8)$, is nothing but the second order Laplace-Beltrami operator. Thus, in this case we can construct other differential operators commuting with $L_{2}$ using higher Laplace operators, i.e. the elements of the center of the universal enveloping algebra of the corresponding Lie algebra. 
In his paper [C6], Cherednik found a representation-theoretic interpretation of Macdonald polynomials for arbitrary Weyl group (and arbitrary values of $k, q$ ), using some generalization of Hecke algebras. This allowed him to give a very elegant construction of the large family of commuting difference operators, for which the Macdonald polynomials are the eigenfunctions. As an application of his technique, he gave an elegant uniform proof of Macdonald's inner product conjectures.

In these lectures we will give an exposition of Cherednik's method. The emphasis will not be on the proof of Macdonald's inner product identities themselves, but rather on the understanding of the algebraic structure hidden behind these polynomials. Once we understand this structure, the proof of the inner product identities is not too difficult. Moreover, the same technique can be used to prove some other properties of these polynomials, such as various symmetry and integrality properties, but this will not be covered in these lectures.

Finally, we note that there is also another representation-theoretic interpretations of Macdonald polynomials, which also gives a natural proof of many of their properties. This approach was developed in a series of papers of Pavel Etingof and the author (see [EK1], [EK2], [EFK]). It is based on the representation theory of the Lie algebra $\mathfrak{s l}_{n}$, or the corresponding quantum group. However, this approach works only for the polynomials associated with the symmetric group and fails for other Weyl groups. The relation between this construction and Cherednik's approach is still unknown.

\section{LeCture 1: Commuting FAmilies of DifFERENTIAL OPERATORs, JaCOBi POLYNOMIALS AND HECKE ALGEBRAS}

This lecture is of an introductory nature. Here we will illustrate the main idea of this course: for certain families of special functions (Jacobi polynomials, Macdonald polynomials), there exist algebraic structures hidden behind them which give a natural explanation of many interesting properties of these polynomials. An example of such properties is the eigenfunctions of a large family of commuting differential (difference) operators. For simplicity, we start with the classical (differential) case, which is more geometrical. In fact, complete proofs for the differential case are more difficult than for the difference one, but since we are not giving proofs in this lecture, the differential case is quite transparent.

We start with a brief survey of the theory of Jacobi polynomials following the papers of Heckman and Opdam [HO], [H1], [O1], [O2]. We do not give any proofs; unless otherwise stated, all the proofs can be found in the above mentioned papers of Heckman and Opdam (though some of the results had been known before). Let $V$ be a vector space over $\mathbb{C}$, and let $R \subset V$ be a (reduced, irreducible) root system of $\operatorname{rank} n=\operatorname{dim} V$. We denote by $S[V]$ the symmetric algebra of $V$, i.e. the algebra of polynomial functions on $V^{*}$. For every $p \in S[V]$ we denote by $\partial_{p}$ the corresponding differential operator with constant coefficients on $V$. We use the standard notations ( , ), $R^{+}, \alpha_{i}, Q, P, W \ldots$ for the inner product in $V$, positive roots, basis of simple roots, root lattice, weight lattice, Weyl group, etc. A reader who does not feel quite comfortable with the root systems can always assume that we are working only with the root system $A_{n-1}$, in which case all the notions above can be described very explicitly as follows:

$V=\left\{v \in \mathbb{C}^{n} \mid v_{1}+\cdots+v_{n}=0\right\}$

inner product: $(a, b)=\sum a_{i} b_{i}$ 
root system: $R=\left\{\varepsilon_{i}-\varepsilon_{j}\right\}_{i \neq j}$, where $\varepsilon_{i}=(0, \ldots, 1,0, \ldots, 0)$ ( 1 in the $i$ th place), $R^{+}=\left\{\varepsilon_{i}-\varepsilon_{j}\right\}_{i<j}$

simple roots: $\alpha_{i}=\varepsilon_{i}-\varepsilon_{i+1}, i=1, \ldots, n-1$

Weyl group: $W=S_{n}$ acts on $V$ by permutation of components; simple reflections $s_{i}$ are transpositions $(i, i+1)$.

root lattice $Q=\left\{\lambda \in \mathbb{Z}^{n} \mid \sum \lambda_{i}=0\right\}$

weight lattice: $P \simeq \mathbb{Z}^{n} / \mathbb{Z}(1, \ldots, 1)$

dominant weights: $P^{+}=\left\{\lambda \in \mathbb{Z}^{n} \mid \lambda_{1} \geq \cdots \geq \lambda_{n}\right\} / \mathbb{Z}(1, \ldots, 1)$

We write $\lambda \leq \mu$ if $\mu-\lambda \in Q_{+}$. Let $\mathbb{C}[P]$ be the group algebra of the weight lattice; it is spanned by the formal exponentials $e^{\lambda}, \lambda \in P$. We can interpret them as functions on $V$ by $e^{\lambda}(v)=e^{(\lambda, v)}$. Again, for the root system $A_{n-1}$ the algebra $\mathbb{C}[P]^{W}$ can be identified with the algebra $\mathbb{C}\left[x_{1}^{ \pm 1}, \ldots, x_{n}^{ \pm 1}\right] /\left(x_{1} \ldots x_{n}=1\right)$, and thus we essentially recover the classical theory of symmetric polynomials discussed in Lecture 0

We will denote by $\mathbb{C}[P]\left(e^{\alpha}-1\right)^{-1}$ the ring of functions on $V$ which have the form $f / g$, where $f, g \in \mathbb{C}[P]$ and $g$ is a product of factors of the form $e^{\alpha}-1, \alpha \in R$.

Let us fix for every $\alpha \in R$ a number $k_{\alpha} \in \mathbb{Z}_{+}$such that $k_{w(\alpha)}=k_{\alpha}$ for every $w \in$ $W$, and define the following $W$-invariant differential operator in $V$ (the CalogeroSutherland-Olshanetsky-Perelomov operator):

$$
L_{2}=\Delta-\sum_{\alpha \in R^{+}} k_{\alpha}\left(k_{\alpha}-1\right) \frac{(\alpha, \alpha)}{\left(e^{\alpha / 2}-e^{-\alpha / 2}\right)^{2}},
$$

where $\Delta$ is the Laplace operator. For the root system $A_{n-1}$ this operator coincides with the Schrödinger operator (0.8), considered in [Su]. The generalization above was suggested by Olshanetsky and Perelomov (see [OP] and references therein).

It was shown in $[\mathrm{CRM}]$ that in the $A_{n}$ case the operator $L_{2}$ defines a completely integrable system, i.e. there are $n$ algebraically independent differential operators commuting with $L_{2}$ and with each other. The following theorem generalizes the complete integrability to arbitrary root systems.

Theorem 1.1 (Heckman, Opdam). Let $\mathcal{D}$ be the ring of differential operators on $V$ with coefficients from the ring $\mathbb{C}[P]\left(e^{\alpha}-1\right)^{-1}$. Let $\mathbb{D}=\{\partial \in \mathcal{D} \mid \partial$ is $W$-invariant, $\left.\left[\partial, L_{2}\right]=0\right\}$. Then there exists "Harish-Chandra isomorphism" $\gamma: \mathbb{D} \simeq(S[V])^{W}$ such that for a homogeneous $p, \gamma^{-1}(p)=\partial_{p}+$ lower order operators. In particular, $\gamma\left(L_{2}\right)=\sum v_{i}^{2}, v_{i}$ being an orthonormal basis in $V$.

Moreover, for most root systems (in particular, for $A_{n}, B_{n}, D_{n}$ with $n \geq 4$ ) it is shown in [OOS] that under suitable restrictions the Sutherland operator, its rational and elliptic analogues (with $\frac{1}{\sinh ^{2} x}$ replaced by $\frac{1}{x^{2}}$ and $\wp(x)$, respectively), and their modifications are the only differential operators of the form $\Delta+V(h)$ which satisfy this complete integrability property.

Remark. It is relatively easy to construct $\gamma$ and show that it is injective (and thus, $\left.\mathbb{D} \subset(S[V])^{W}\right)$; the difficult part is to prove that $\gamma$ is surjective.

This theorem naturally gives rise to the following questions:

1. What are the eigenfunctions of $\partial \in \mathbb{D}$ and what are their properties?

2. Why is it that $\mathbb{D} \simeq(S[V])^{W}$ ? Is there any natural explanation for this fact that leads to a construction of $\gamma^{-1}$ ? 
3. Is it possible to extend $\gamma^{-1}$ from symmetric to all polynomials, that is, to construct for every $v \in V$ a differential operator $D_{v}$ such that (1) $D_{v}$ commute and (2) $w D_{v} w^{-1}=D_{w v}$ for every $w \in W$ in such a way that $\gamma^{-1}(v)=D_{v}$ ?

We will try to answer these questions. Let us start with the last one. Here the answer is obviously "no". It is so even in the case of the root system of type $A_{1}\left(\mathfrak{s l}_{2}\right.$ case), where $L_{2}=\frac{d^{2}}{d x^{2}}+C \frac{1}{\sinh ^{2} x}$, which obviously is not a square of any first-order differential operator.

This is analogous to the definition of the Dirac operator in physics. Recall that the Dirac operator was introduced as an attempt to find a square root of the Laplace operator. Such a square root does not exist in the class of scalar-valued differential operators. However, if one considers differential operators with values in the Clifford algebra, then such a square root does exist, and it is called the Dirac operator.

Similar construction is possible here, and the corresponding algebraic structure - parallel to that of Clifford algebra - is a degenerate affine Hecke algebra. Before describing it, let us slightly reformulate the problem.

Let

$$
\begin{gathered}
\delta^{k}=\prod_{\alpha \in R^{+}}\left(e^{\alpha / 2}-e^{-\alpha / 2}\right)^{k_{\alpha}}, \\
\rho_{k}=\frac{1}{2} \sum_{\alpha \in R^{+}} k_{\alpha} \alpha .
\end{gathered}
$$

Now, define

$$
M_{2}=\delta^{-k}\left(L_{2}-\left(\rho_{k}, \rho_{k}\right)\right) \delta^{k}
$$

\section{Proposition 1.2.}

$$
M_{2}=\Delta-\sum_{\alpha \in R^{+}} k_{\alpha} \frac{1+e^{\alpha}}{1-e^{\alpha}} \partial_{\alpha} .
$$

Proposition 1.3. $\quad$ 1. $M_{2}$ preserves the space $\mathbb{C}[P]^{W}$ of Weyl group invariant polynomials, and so do all the operators from $\delta^{-k} \mathbb{D} \delta^{k}$.

2. Let $m_{\lambda}=\sum_{\mu \in W \lambda} e^{\mu}, \lambda \in P_{+}$be the basis of orbit-sums in $\mathbb{C}[P]^{W}$. Then the action of $M_{2}$ is triangular in this basis:

$$
M_{2} m_{\lambda}=\left(\lambda, \lambda+2 \rho_{k}\right) m_{\lambda}+\sum_{\mu<\lambda} c_{\lambda \mu} m_{\mu}
$$

Thus, we can restrict ourselves to considering only the action of $M_{2}$ on $\mathbb{C}[P]^{W}$ and study only symmetric polynomial eigenfunctions.

Definition. Jacobi polynomials $J_{\lambda} \in \mathbb{C}[P]^{W}, \lambda \in P_{+}$are defined by the following conditions:

1. $J_{\lambda}=m_{\lambda}+$ lower order terms.

2. $M_{2} J_{\lambda}=\left(\lambda, \lambda+2 \rho_{k}\right) J_{\lambda}$.

It is easy to show that these conditions define $J_{\lambda}$ uniquely. These polynomials have a number of interesting properties; for example, they are orthogonal with respect to a certain inner product which will be discussed in the next lecture. For special values of $k_{\alpha}$ these polynomials can be interpreted as zonal spherical 
functions on certain Riemannian symmetric spaces for the Lie group $G$ associated with the root system $R$ (see [H1]). In the case of the root system of type $A_{n}$, the polynomials $J_{\lambda}$ are known under the name "Jack polynomials" and can be interpreted as vector-valued zonal spherical functions for arbitrary $k \in \mathbb{Z}_{+}$(see [EFK]).

Let us return to questions 2 and 3 above: is it possible to introduce commuting operators $D_{v}, v \in V$ such that $w D_{v} w^{-1}=D_{w v}$ for any $w \in W$, and $M_{2}=\sum D_{v_{i}}^{2}+$ const, $v_{i}$ an orthonormal basis in $V$ ? As we have seen before, if $D_{v}$ are differential operators acting on scalar functions, then the answer is "no". Therefore, it is natural to try solving this problem using some larger class of operators. This can be done in two ways; in fact, they are closely related and can be considered as special cases of a general approach (see [C3], [C5]), but we will not go into details here.

1. We can let $D$ be matrix-valued differential operators: it is possible to introduce $D_{v}$ which act on functions with values in some vector space $E$, and a linear map $E \rightarrow \mathbb{C}$ such that any matrix-valued differential operator which is obtained as a symmetric polynomial of $D_{v_{i}}$ can be pushed forward to some scalar differential operator. In this way one can get the commuting family of differential operators discussed above from the symmetric polynomials in $D_{v_{i}}$. This approach was studied in detail by Matsuo ([Ma]), who considered $E=\mathbb{C}[W]$. This construction will not be used in these lectures.

2 . We can let $D$ be scalar valued operators involving not only differentiation but also the action of the Weyl group (which acts on functions by permuting arguments). These operators are not local; however, it is possible to choose them in such a way that symmetric polynomials of $D_{v_{i}}$ preserve the subspace $\mathbb{C}[P]^{W}$ of Weyl group invariant polynomials, and their restrictions to $\mathbb{C}[P]^{W}$ coincide with certain differential operators. In particular, $\left.\left(\sum D_{v_{i}}^{2}\right)\right|_{\mathbb{C}[P]^{W}}=$ $M_{2}$.

We will be most interested in this last approach. In the next lectures we will use it in the difference case to get a difference analogue of this commuting family of differential operators, study their polynomial eigenfunctions (Macdonald polynomials), and prove Macdonald's inner product identities. In this lecture we will illustrate the ideas with a "baby example". Namely, let us consider the rational degeneration of the above differential operators. Introduce a rescaling operator $A_{t}$ by $\left(A_{t} f\right)(v)=f(t v)$. Consider $L_{2}(t)=t^{-2} A_{t}^{-1} L_{2} A_{t}$. Then it is easy to see that as $t \rightarrow 0, L_{2}(t)$ has a limit, which we will call $L_{2}^{r a t}$ :

$$
L_{2}^{r a t}(v)=\Delta-\sum_{\alpha \in R^{+}} k_{\alpha}\left(k_{\alpha}-1\right) \frac{(\alpha, \alpha)}{(\alpha, v)^{2}} .
$$

Similarly, we can get the rational degeneration of $M_{2}$ :

$$
M_{2}^{r a t}(v)=\Delta-\sum_{\alpha \in R^{+}} k_{\alpha} \frac{1}{(\alpha, v)} \partial_{\alpha}
$$

Consider the $\mathfrak{s l}_{n}$ case, that is, the root system of type $A_{n-1}$. Then $V \subset \mathbb{C}^{n}$, and we can identify functions on $V$ with functions on $\mathbb{C}^{n}$ which are invariant with respect to the translations $\left(x_{1}, \ldots, x_{n}\right) \mapsto\left(x_{1}+c, \ldots, x_{n}+c\right)$. In this case all $k_{\alpha}$ 
are equal: $k_{\alpha}=k$ and $M_{2}^{r a t}$ takes the form

$$
M_{2}^{r a t}=\sum \partial_{i}^{2}+2 k \sum_{i<j} \frac{1}{x_{i}-x_{j}}\left(\partial_{i}-\partial_{j}\right)
$$

where $\partial_{i}=\frac{\partial}{\partial x_{i}}$.

We will show how one can obtain an expression for $M_{2}^{\text {rat }}$ of the form $M_{2}^{\text {rat }}=$ $\sum D_{i}^{2}$ using so-called differential-difference operators introduced by Dunkl (see $[\mathrm{H} 2])$. Let $b_{i j}$ be the following operator:

$$
b_{i j} f=\frac{s_{i j} f-f}{x_{i}-x_{j}}
$$

where $s_{i j}$ acts on functions of $x_{1}, \ldots, x_{n}$ by permutation of arguments: $x_{i} \leftrightarrow x_{j}$. Note that $b_{i j}$ preserves the space $\mathbb{C}\left[x_{1}, \ldots, x_{n}\right]$, since $x^{n} y^{m}-x^{m} y^{n}$ is divisible by $x-y$. Also, it is easy to see that $w b_{i j} w^{-1}=b_{w(i) w(j)}$ for any $w \in S_{n}$.

Define the rational Dunkl operators by

$$
D_{i}=\partial_{i}-k \sum_{j \neq i} b_{i j}
$$

(sometimes they are also called local Dunkl operators, as opposed to the operators with trigonometric coefficients which are called global).

Theorem 1.4. $\quad$ 1. $w D_{i} w^{-1}=D_{w(i)}$ for any $w \in S_{n}$.

2. $\left[D_{i}, D_{j}\right]=0$.

Proof. (1) is trivial. Let us prove (2). Obviously, $\left[D_{i}, D_{j}\right]$ contains only terms linear in $k$ and quadratic in $k$. Since $\left[\partial_{i}, b_{l m}\right]=0$ if $i \neq l, m$, the term linear in $k$ is equal to $-k\left(\left[\partial_{i}, b_{j i}\right]-\left[\partial_{j}, b_{i j}\right]\right)$. Since $b_{i j}=-b_{j i}$, this equals $k\left[\partial_{i}+\partial_{j}, b_{i j}\right]=0$.

Standard arguments show that in order for the term quadratic in $k$ to vanish it is necessary and sufficient that $b_{i j}$ satisfy the classical Yang-Baxter equation:

$$
\left[b_{12}, b_{13}\right]+\left[b_{12}, b_{23}\right]+\left[b_{13}, b_{23}\right]=0 \text {. }
$$

This can be proved by direct calculation, which is rather boring. We will show another way to prove it later.

For every operator of the form $D=\sum_{w \in S_{n}} D_{w} w$, where $D_{w}$ is a differential operator with rational coefficients, define the associated differential operator by

$$
\operatorname{Res}\left(\sum D_{w} w\right)=\sum D_{w}
$$

Note that if $D$ preserves the space of symmetric polynomials, then so does Res $D$, and $\left.D\right|_{\mathbb{C}[P]^{W}}=\left.\operatorname{Res} D\right|_{\mathbb{C}[P]^{W}}$.

Theorem 1.5. $\sum D_{i}^{2}$ is $S_{n}$-invariant, preserves $\mathbb{C}\left[x_{1}, \ldots, x_{n}\right]^{S_{n}}$ and $\operatorname{Res}\left(\sum D_{i}^{2}\right)$ $=M_{2}^{r a t}$.

Proof. Invariance of $\left(\sum D_{i}^{2}\right)$ and the fact that it preserves $\mathbb{C}\left[x_{i}\right]^{S_{n}}$ follow from the previous theorem. To calculate $\operatorname{Res}\left(\sum D_{i}^{2}\right)$, write

$$
\sum D_{i}^{2}=\sum_{i}\left(\partial_{i}^{2}-k \sum_{j \neq i}\left(\partial_{i} b_{i j}+b_{i j} \partial_{i}\right)+4 k^{2} \sum_{\substack{j \neq i \\ l \neq i}} b_{i j} b_{i l}\right) .
$$


Since $\left.b_{i j}\right|_{\mathbb{C}[x]^{S_{n}}}=0$ (this is the crucial step!), we have

$$
\operatorname{Res} \sum D_{i}^{2}=\operatorname{Res}\left(\sum_{i}\left(\partial_{i}^{2}-k \sum_{j \neq i} b_{i j} \partial_{i}\right)\right) \text {. }
$$

It is easy to check that

$$
b_{i j} \partial_{i}=\left(x_{i}-x_{j}\right)^{-1}\left(s_{i j}-1\right) \partial_{i}=\left(x_{i}-x_{j}\right)^{-1}\left(\partial_{j} s_{i j}-\partial_{i}\right)
$$

and thus

$$
\operatorname{Res}\left(\sum D_{i}^{2}\right)=\sum_{i}\left(\partial_{i}^{2}-k \sum_{j \neq i}\left(x_{i}-x_{j}\right)^{-1}\left(\partial_{j}-\partial_{i}\right)\right)=M_{2}^{r a t} .
$$

Corollary. $M_{2}^{\text {rat }}$ can be included in a commutative family of symmetric differential operators $M_{1}^{\text {rat }}=\sum \partial_{i}, M_{2}^{\text {rat }}, \ldots, M_{n}^{\text {rat }}$ with coefficients from $\mathbb{C}\left[x_{1}, \ldots, x_{n}\right]\left(x_{i}-x_{j}\right)^{-1}$.

Proof. Take $M_{r}^{\text {rat }}=\operatorname{Res} \sum D_{i}^{r}$.

Thus, we have proved in this baby example an analogue of the complete integrability of Theorem 1.1 and given an explicit construction of these differential operators. However, even in this case there remain some questions:

1. How did we get the expressions for $D_{i}$ and $b_{i j}$ ? Is there a way to guess them?

2 . Why do $b_{i j}$ satisfy the Yang-Baxter equation?

The answer to these questions is that there exists a simple algebraic construction which allows us to get the expressions for $b_{i j}$ as well as their properties without any calculation. This is the degenerate affine Hecke algebra.

Definition. The degenerate affine Hecke algebra for the root system $A_{n-1}$ is the algebra $H_{n}^{\prime}$ over $\mathbb{C}$ spanned by its two subalgebras $\mathbb{C}\left[S_{n}\right]$ and $\mathbb{C}\left[x_{1}, \ldots, x_{n}\right]$ with the relations

$$
\begin{gathered}
x_{i+1} s_{i}-s_{i} x_{i}=s_{i} x_{i+1}-x_{i} s_{i}=h, \\
x_{i} s_{j}=s_{j} x_{i} \quad \text { if } i \neq j, j+1,
\end{gathered}
$$

where $h \in \mathbb{C}$ is a fixed constant, and $s_{i}=s_{i, i+1}, i=1 \ldots n-1$ are the standard generators of $S_{n}$.

For $h=0$ this algebra coincides with the semidirect product $\mathbb{C}\left[S_{n}\right] \ltimes \mathbb{C}[x]$ (for brevity, we write $\mathbb{C}[x]$ for $\left.\mathbb{C}\left[x_{1}, \ldots, x_{n}\right]\right)$. Therefore, we can consider $H_{n}^{\prime}$ as a deformation of $\mathbb{C}\left[S_{n}\right] \ltimes \mathbb{C}[x]$.

Theorem 1.6. $H_{n}^{\prime}=\mathbb{C}\left[S_{n}\right] \cdot \mathbb{C}[x]=\mathbb{C}[x] \cdot \mathbb{C}\left[S_{n}\right]$; that is, every $g \in H_{n}^{\prime}$ can be uniquely written in either of the following forms:

$$
g=\sum_{w \in S_{n}} p_{w}(x) w=\sum_{w \in S_{n}} w q_{w}(x)
$$

where $p_{w}, q_{w} \in \mathbb{C}[x]$.

We refer the reader to [C5] and references therein for the proof. Note that it is obvious that every element can be written in either of the forms in (1.12); the difficult part is to prove the uniqueness. 
Remark. Of course, a degenerate affine Hecke algebra can be defined for any root system, and the analogue of Theorem 1.6 is also true; see [C5].

Now, let $E$ be any module over $S_{n}$. Define the induced module $\widehat{E}=\operatorname{Ind}_{\mathbb{C}\left[S_{n}\right]}^{H_{n}^{\prime}} E$. It follows from the theorem above that as a vector space (and, moreover, as a $\mathbb{C}[x]$ module), $\widehat{E} \simeq E \otimes \mathbb{C}[x]$. In particular, let us take $E=\mathbb{C}$ with the trivial action of $S_{n}$. Then $\widehat{E} \simeq \mathbb{C}[x]$, and we get the following proposition:

Proposition 1.7. There exists a unique action of $H_{n}^{\prime}$ on $\mathbb{C}[x]$ such that

1. $p \in \mathbb{C}[x] \subset H_{n}^{\prime}$ acts by multiplication by $p$.

2. $\hat{s}_{i} 1=1$, where $\hat{s}$ denotes the action of an element $s \in S_{n} \subset H_{n}^{\prime}$ on $\mathbb{C}[x]$.

Proposition 1.8. In the above defined representation,

$$
\hat{s}_{i}=s_{i}+h b_{i, i+1},
$$

where $s_{i}$ is the usual action of $S_{n}$ on $\mathbb{C}[x]$ (by permutation of $x_{i}$ ), and $b_{i j}$ is defined by (1.8).

Proof. It is easy to see that the conditions $\hat{s}_{i} 1=1$ and the commutation relations (1.11) uniquely define the operators $\hat{s}_{i}$ on $\mathbb{C}[x]$. But the $\hat{s}_{i}$ defined by formula (1.13) satisfy both $\hat{s}_{i} 1=1$ (obvious) and (1.11), which can be shown by a rather short explicit calculation.

Thus, we see that the operators $b_{i j}$ which we defined before have a very natural interpretation in terms of the degenerate affine Hecke algebra: they describe the action of $H_{n}^{\prime}$ in the induced representation. This allows us to prove easily the classical Yang-Baxter equation for $b_{i j}$. Indeed, it follows from Propositions 1.7 and 1.8 that the $\hat{s}_{i}$ defined by (1.13) satisfy the braid relations:

$$
\hat{s}_{1} \hat{s}_{2} \hat{s}_{1}=\hat{s}_{2} \hat{s}_{1} \hat{s}_{2} \text {. }
$$

Let us define $R_{12}=s_{1} \hat{s}_{1}, R_{23}=s_{2} \hat{s}_{2}$, and $R_{13}=s_{1} R_{23} s_{1}=s_{2} R_{12} s_{2}$ (check this last identity!). A simple calculation shows that the braid relation for $\hat{s}_{i}$ implies the quantum Yang-Baxter equation for $R_{i j}$ :

$$
R_{12} R_{13} R_{23}=R_{23} R_{13} R_{12} .
$$

Since $R_{i j}=1+h b_{i j}$, it is a standard fact that the quantum Yang-Baxter equation for $R_{i j}$ implies the classical Yang-Baxter equation for $b_{i j}$.

Thus, we have shown that using the notion of the degenerate affine Hecke algebra along with the "Poincare-Birkhoff-Witt theorem", 1.6, we can easily and naturally derive formula (1.9) for $D_{i}$ and prove all the required properties. This is the main idea of this course. In the next lectures we will explain in detail how a similar approach works in the difference case.

Note that the proof of Theorem 1.6 is rather difficult and requires about as many calculations as a straightforward proof of the Yang-Baxter equations, so use of affine Hecke algebras does not allow us to avoid the calculations. The advantage of this approach is that it allows us to organize the calculations in a much clearer way.

Remark. If we want to apply the construction above to the trigonometric case that is, to the original operator $L_{2}$ rather than to its rational degeneration - than we need to make certain changes. Namely, in this case we cannot construct $D_{v}$ which commute and satisfy the relation $w D_{v} w^{-1}=D_{w v}$. However, we can construct 
something very close to it: we can construct $D_{v}$ such that they commute and their commutation relations with $S_{n}$ are given by formula (1.11); in other words, they satisfy the relations of a degenerate affine Hecke algebra. This still allows us to get a commuting family of differential operators, since it is known that $\mathbb{C}[x]^{S_{n}}$ is the center of $H_{n}^{\prime}$.

\section{LECTURE 2: MACDONALD POLYNOMIALS AND DIFFERENCE OPERATORS}

In this lecture we start a systematic study of the difference case. We will not refer to the first lecture (except as a motivation).

In this lecture we define the $q$-analogue of the Jacobi polynomials discussed in Lecture 1. Unless otherwise stated, the results in this lecture are due to Macdonald ([M1], [M2]; see also the exposition in [M6, Chapter VI]).

We begin by fixing the notation. Let $V$ be a finite-dimensional vector space over $\mathbb{R}$ with a positive definite, symmetric, bilinear form $(\cdot, \cdot)$, and let $R \subset V$ be a reduced, irreducible root system. We fix a decomposition of $R$ into positive and negative roots: $R=R^{+} \sqcup R^{-}$and denote by $\alpha_{1}, \ldots, \alpha_{n}$ the basis of simple roots in $R^{+}$. For every root $\alpha$ define its dual root $\alpha^{\vee}=\frac{2 \alpha}{(\alpha, \alpha)}$. Let $Q=\bigoplus \mathbb{Z} \alpha_{i}$ be the root lattice, $Q_{+}=\bigoplus \mathbb{Z}_{+} \alpha_{i}, P=\left\{\lambda \in V \mid\left(\lambda, \alpha_{i}^{\vee}\right) \in \mathbb{Z}\right\}$ the weight lattice, and $P_{+}=\left\{\lambda \in V \mid\left(\lambda, \alpha_{i}^{\vee}\right) \in \mathbb{Z}_{+}\right\}$the cone of dominant integral weights. $P$ has a natural basis of fundamental weights $\omega_{i}:\left(\omega_{i}, \alpha_{j}^{\vee}\right)=\delta_{i j}$. In a similar way, let $Q^{\vee}=\bigoplus \mathbb{Z} \alpha_{i}^{\vee}$ be the coroot lattice, $Q_{+}^{\vee}=\bigoplus \mathbb{Z}_{+} \alpha_{i}^{\vee}, P^{\vee}=\left\{\lambda \in V \mid\left(\lambda, \alpha_{i}\right) \in \mathbb{Z}\right\}$ the coweight lattice, $P_{+}^{\vee}=\left\{\lambda \in V \mid\left(\lambda, \alpha_{i}\right) \in \mathbb{Z}_{+}\right\}$the dominant coweights, and $b_{i}$ the fundamental coweights: $\left(b_{i}, \alpha_{j}\right)=\delta_{i j}$. As usual, we define the highest root $\theta \in R$ by the condition $\theta-\alpha \in Q_{+}$for all $\alpha \in R$. Let $\rho=\frac{1}{2} \sum_{\alpha \in R_{+}} \alpha$; then $\left(\rho, \alpha_{i}^{\vee}\right)=1$, so $\rho \in P$.

For every $\alpha \in R$, let $s_{\alpha}$ be the corresponding reflection, and let $W$ be the Weyl group generated by the $s_{\alpha}$. Then $W$ acts on $P$ and thus on the group algebra $\mathbb{C}[P]$. We denote the subspace of $W$-invariants in $\mathbb{C}[P]$ by $\mathbb{C}[P]^{W}$; elements of $\mathbb{C}[P]^{W}$ will be called "symmetric polynomials".

An example of a basis in $\mathbb{C}[P]^{W}$ is given by the orbit-sums: $m_{\lambda}=\sum_{\mu \in W \lambda} e^{\mu}, \lambda \in$ $P_{+}$. This basis is orthogonal with respect to the following inner product on $\mathbb{C}[P]$ : $\langle f, g\rangle_{0}=\frac{1}{|W|}[f \bar{g}]_{0}$, where the bar involution is defined by $\overline{e^{\lambda}}=e^{-\lambda}$, and []$_{0}$ is the constant term: $\left[\sum a_{\lambda} e^{\lambda}\right]_{0}=a_{0}$.

This basis admits the following generalization. Assigned to every $\alpha \in R$ we have a variable $t_{\alpha}$ subject to the conditions $t_{\alpha}=t_{w(\alpha)}$ (thus, we have at most 2 different $t$ 's). Let $q$ be another independent variable and let $\mathbb{C}_{q, t}=\mathbb{C}\left(q, t_{\alpha}\right)$ be the field of rational functions in $t_{\alpha}, q$. Define the inner product on $\mathbb{C}_{q, t}[P]$ by

$$
\langle f, g\rangle_{q, t}=\frac{1}{|W|}\left[f \bar{g} \Delta_{q, t}\right]_{0}
$$

where the bar involution is extended by $\mathbb{C}_{q, t}$ linearity, and

$$
\Delta_{q, t}=\prod_{\alpha \in R} \prod_{i=0}^{\infty} \frac{1-q^{2 i} e^{\alpha}}{1-t_{\alpha}^{2} q^{2 i} e^{\alpha}}=\Delta_{q, t}^{+} \overline{\Delta_{q, t}^{+}},
$$

where

$$
\Delta_{q, t}^{+}=\prod_{\alpha \in R^{+}} \prod_{i=0}^{\infty} \frac{1-q^{2 i} e^{\alpha}}{1-t_{\alpha}^{2} q^{2 i} e^{\alpha}}
$$


Both $\Delta$ and $\Delta^{+}$should be considered as Laurent series in $q, t_{\alpha}$ with coefficients from $\mathbb{C}[P]$; then the inner product also takes values in the Laurent series. It is easy to see that this inner product is non-degenerate and $W$-invariant. If $t_{\alpha}=1$ for all $\alpha$, then this inner product coincides with previously defined $\langle\cdot, \cdot\rangle_{0}$.

Theorem 2.1(Macdonald). There exists a unique family of symmetric polynomials $P_{\lambda} \in \mathbb{C}_{q, t}[P]^{W}, \lambda \in P_{+}$satisfying the following conditions:

1. $P_{\lambda}=m_{\lambda}+\sum_{\mu<\lambda} a_{\lambda \mu} m_{\mu}$,

2. $\left\langle P_{\lambda}, P_{\mu}\right\rangle_{q, t}=0$ if $\lambda \neq \mu$.

These polynomials are called Macdonald polynomials (see [M1], [M2]), and form a basis in $\mathbb{C}_{q, t}[P]^{W}$. Note that the theorem above is not trivial: since $<$ is not a complete order, one cannot get $P_{\lambda}$ by orthogonalization of $m_{\lambda}$.

Remark. Our notation differs slightly from the original notation of Macdonald: what we denote by $q, t_{\alpha}$ in his notations would be $q^{1 / 2}, t_{\alpha}^{1 / 2}$.

Examples. 1 . If $t_{\alpha}=1$, then $P_{\lambda}=m_{\lambda}$ does not depend on $q$.

2. If $t_{\alpha}=q$ for all $\alpha$, then $P_{\lambda}=\chi_{\lambda}$ are the Weyl characters.

3 . If $q, t \rightarrow 1$ in such a way that $t_{\alpha}=q^{k_{\alpha}}, k_{\alpha} \in \mathbb{Z}_{+}$fixed, then $P_{\lambda} \rightarrow J_{\lambda}$, where $J_{\lambda}$ are the Jacobi polynomials defined in the previous lecture as eigenfunctions of some differential operator $M_{2}$. Indeed, we have $\Delta_{q, t}^{+} \rightarrow \delta^{k}$, and it suffices to check that $M_{2}$ is self-adjoint with respect to the inner product $\langle f, g\rangle=$ $\frac{1}{|W|}\left[f \bar{g}(\delta \bar{\delta})^{k}\right]_{0}=\left\langle f \delta^{k}, g \delta^{k}\right\rangle_{0}$. This is equivalent to the fact that $L_{2}$ is selfadjoint with respect to $\langle,\rangle_{0}$, which is obvious.

4. If $q=0, t_{\alpha}^{2}=1 / p$, where $p$ is prime, then $P_{\lambda}$ can be interpreted as zonal spherical functions on certain symmetric spaces for $p$-adic groups.

5. For the root system $A_{1}$ Macdonald polynomials coincide with the so-called $q$-ultraspherical polynomials ([AI]), which are a special case of Askey-Wilson polynomials $([\mathrm{AW}])$. More generally, Macdonald polynomials for the nonreduced root system $B C_{1}$ are precisely continuous $q$-Jacobi polynomials, which are again a special case of Askey-Wilson polynomials.

For simplicity, we only consider in these lectures the case where $t_{\alpha}=q^{k_{\alpha}}$ for some $k_{\alpha} \in \mathbb{Z}_{+}$. It is not an important restriction: all the results we prove can be generalized to the case where $q, t$ are independent variables. However, the case where $t_{\alpha}=q^{k_{\alpha}}$ is easier from the technical point of view; for example, formula (2.2) takes the form

$$
\Delta_{q, t}=\prod_{\alpha \in R} \prod_{i=0}^{k_{\alpha}-1}\left(1-q^{2 i} e^{\alpha}\right)
$$

thus allowing us to avoid infinite products. We will use the notations $\langle,\rangle_{k}, \Delta_{k}$ for $\langle,\rangle_{q, t}, \Delta_{q, t}$, etc. Also, it will be convenient to denote $\rho_{k}=\frac{1}{2} \sum_{\alpha \in R^{+}} k_{\alpha} \alpha$. We always consider $q$ as a formal variable; the results also hold if $q$ is a complex number provided that it is not a root of unity.

As in the differential case, for some special values of $k$ the Macdonald polynomials can be interpreted as zonal spherical functions on certain $q$-symmetric spaces associated with the group $G$ (see $[\mathrm{N}])$.

Sketch of proof of Theorem 2.1. To prove the theorem, it suffices to find an operator $D: \mathbb{C}_{q}[P]^{W} \rightarrow \mathbb{C}_{q}[P]^{W}$ such that 
1. $D m_{\lambda}=\sum_{\substack{\mu \in P_{+} \\ \mu \leq \lambda}} c_{\lambda \mu} m_{\mu}$.

2. $c_{\lambda \lambda}$ are distinct.

3. $D$ is self-adjoint with respect to the inner product $\langle,\rangle_{\text {q.t }}$.

Let us construct such an operator.

Definition. A weight $\lambda \in P^{+}$is called minuscule if $0 \leq\left(\lambda, \alpha^{\vee}\right) \leq 1$ for every $\alpha \in R^{+}$.

It is easy to see that a weight $\lambda \neq 0$ can be minuscule only if $\lambda$ is one of the fundamental weights. Indeed, let $\phi$ be the highest root for the root system $R^{\vee}$; then $\phi=\sum n_{i} \alpha_{i}^{\vee}, n_{i}>0$. Thus, $(\lambda, \phi) \leq 1$ implies that $\lambda=0$ or $\lambda=\omega_{r}$ for some $r$ such that $n_{r}=1$. The following lemma describes the set of minuscule weights.

Lemma 2.2. (see $[\mathrm{B}],[\mathrm{V}]$ ) The set of all minuscule weights is a system of representatives for $P / Q$ : every $\lambda \in P$ can be uniquely written in the form $\lambda=b+\alpha$ for some minuscule weight $b$ and $\alpha \in Q$.

This implies that there are no non-zero minuscule weights for the root systems $E_{8}, F_{4}, G_{2}$ and that the number of minuscule weights is always less than or equal to the rank of the root system with equality only for $A_{n}$.

Let $\pi \in P^{\vee}$ be a minuscule coweight: $0 \leq(\pi, \alpha) \leq 1$ for all $\alpha \in R^{+}$. Define $T_{\pi}$ : $\mathbb{C}_{q}[P] \rightarrow \mathbb{C}_{q}[P]$ by $T_{\pi}\left(e^{\lambda}\right)=q^{2(\lambda, \pi)} e^{\lambda}$ (this requires adding appropriate fractional powers of $q$ to $\mathbb{C}_{q}$ ). Let $D_{\pi}$ be defined by

$$
D_{\pi}(f)=\sum_{w \in W} w\left(\frac{T_{\pi}\left(\Delta^{+} f\right)}{\Delta^{+}}\right)=\sum_{w \in W} w\left(\prod_{\substack{\alpha \in R^{+} \\(\alpha, \pi)=1}} \frac{1-q^{2 k_{\alpha}} e^{\alpha}}{1-e^{\alpha}} T_{\pi}(f)\right) .
$$

Let us prove that $D_{\pi} f \in \mathbb{C}_{q}[P]^{W}$ for every $f \in \mathbb{C}_{q}[P]$. It is obvious from (2.4) that $D_{\pi} f$ is a $W$-invariant rational function with poles only at $e^{\alpha}-1=0$ and all the poles are simple. Thus, $\delta D_{\pi} f$ (where $\delta=\prod_{\alpha \in R^{+}}\left(e^{\alpha / 2}-e^{-\alpha / 2}\right)$ is the Weyl denominator) is a $W$-anti-invariant element of $\mathbb{C}_{q}[P]$. It is well known that this implies that $D_{\pi} f \in \mathbb{C}_{q}[P]^{W}$.

The triangularity condition (1) above can be easily verified by direct calculation, which also shows that $c_{\lambda \lambda}=q^{2\left(\pi, \rho_{k}\right)} \sum_{w \in W} q^{2\left(\pi, w\left(\lambda+\rho_{k}\right)\right)}$ (to show this one needs the identity $\left.\rho_{k}-w\left(\rho_{k}\right)=\sum_{\alpha \in R^{+} \cap w^{-1} R^{-}} k_{\alpha} \alpha\right)$. It is also easy to deduce from (2.4) and the definition of the inner product that $D_{\pi}$ is self-adjoint.

Finally, one can check that for all root systems having non-zero minuscule coweights, with the exception of $D_{n}$, there exists a minuscule coweight $\pi$ such that the corresponding eigenvalues $c_{\lambda \lambda}$ are distinct. For $D_{n}$ it is not so, but there exists a linear combination of operators $D_{\pi}$ corresponding to minuscule coweights such that the eigenvalues are distinct. This proves the theorem for all cases where non-zero minuscule coweights exist, that is, for all cases except $E_{8}, F_{4}, G_{2}$.

The above proof used the fact that $\pi$ is a minuscule coweight: otherwise, $D_{\pi} f$ could have products of the form $\left(1-e^{\alpha}\right)\left(1-q^{2} e^{\alpha}\right) \ldots$ in the denominator. Thus, this proof fails for the root systems $E_{8}, F_{4}, G_{2}$. This, however, can be fixed, but this requires a certain ingenuity; we refer the reader to the original papers of Macdonald.

The operators $D_{\pi}$ have some interesting properties. Here is one of them:

Proposition 2.3. If $\pi_{1}, \pi_{2}$ are minuscule coweights, then $D_{\pi_{1}}, D_{\pi_{2}}$ commute. 
The proof of this proposition is straightforward.

Example. Let $R \subset V$ be of type $A_{n-1}$; we identify $V$ with a subspace in $\mathbb{R}^{n}$ given by the condition $\sum \lambda_{i}=0$. Then $e^{\lambda} \mapsto x_{1}^{\lambda_{1}} \ldots x_{n}^{\lambda_{n}}$, where $\lambda=\left(\lambda_{1}, \ldots, \lambda_{n}\right) \in$ $V \subset \mathbb{R}^{n}$, gives an isomorphism of $\mathbb{C}_{q}[P]$ with the space of homogeneous polynomials in $x_{i}^{ \pm 1}$ of degree zero. In this case all the fundamental weights (=coweights) $\omega_{1}, \ldots, \omega_{n-1}$ are minuscule, and the corresponding operators take the form

$$
D_{r}=\sum_{w} w\left(\prod_{\substack{i, j \\ i \leq r<j}} \frac{x_{i}-q^{2 k} x_{j}}{x_{i}-x_{j}} T_{1 \ldots r}\right)=r !(n-r) ! \sum_{\substack{I \subset\{1 \ldots n\} \\|I|=r}}\left(\prod_{\substack{i \in I \\ j \notin I}} \frac{x_{i}-q^{2 k} x_{j}}{x_{i}-x_{j}}\right) T_{I},
$$

where $T_{I}=\prod_{i \in I} T_{i},\left(T_{i} f\right)\left(x_{1}, \ldots, x_{n}\right)=f\left(x_{1}, \ldots, q^{2} x_{i}, \ldots, x_{n}\right)$.

In this example, we have constructed a commuting family of difference operators, and the number of independent operators is equal to the rank of the root system. This family is the natural quantum analogue of the commuting families of differential operators considered in Lecture 1. However, this analogy fails for other root systems since the number of difference operators we get from the minuscule coweights is in general less than the rank of the root system.

This discrepancy can be resolved as follows: for any root system there exists a commuting family of difference operators, and the number of independent operators is equal to the rank of the root system, but not all of them can be obtained from minuscule coweights. One of the main goals of the subsequent lectures will be construction of these difference operators based on the representation theory of affine Hecke algebras. In general, explicit expressions for these operators are rather complicated, which makes them impossible to guess; the fact that the operators corresponding to the minuscule coweights can be written by such a simple formula as in (2.4) is a lucky exception. Van Diejen ([D]) also found explicit formulas for these operators for the non-reduced root system $B C_{n}$ and, even more generally, for the 5-parameter family of Koornwinder polynomials ([Ko2]), which generalize both Macdonald polynomials for $B C_{n}$ and Askey-Wilson polynomials; however, these formulas are rather complicated.

Now we can formulate Macdonald's inner product identity.

Theorem 2.4. (Macdonald's inner product identity)

$$
\begin{aligned}
\left\langle P_{\lambda}, P_{\lambda}\right\rangle_{k} & =\prod_{\alpha \in R^{+}} \prod_{i=1}^{k_{\alpha}-1} \frac{1-q^{2\left(\alpha^{\vee}, \lambda+\rho_{k}\right)+2 i}}{1-q^{2\left(\alpha^{\vee}, \lambda+\rho_{k}\right)-2 i}} \\
& =\prod_{\alpha \in R^{+}} \prod_{i=1}^{k_{\alpha}-1} \frac{\left[\left(\alpha^{\vee}, \lambda+\rho_{k}\right)+i\right]}{\left[\left(\alpha^{\vee}, \lambda+\rho_{k}\right)-i\right]},
\end{aligned}
$$

where $[n]=\frac{1-q^{2 n}}{1-q^{2}}$.

This formula was conjectured by Macdonald, who proved it for the root systems of type $A$ (see [M6, Chapter VI, Example 1]). Also, it was proven for the root system $B C_{1}$ (see $[\mathrm{AW}]$ ). The first proof for an arbitrary root system was given by Cherednik; we will give this proof in the following lectures. 
Examples. 1. Let $k_{\alpha}=1$. Then $P_{\lambda}=\chi_{\lambda}$ are the Weyl characters, and (2.6) reduces to $\left\langle\chi_{\lambda}, \chi_{\lambda}\right\rangle_{1}=1$, which is well-known.

2. Let $\lambda=0$. Then $P_{\lambda}=1$, and (2.6) reduces to

$$
\frac{1}{|W|}\left[\Delta_{q, t}\right]_{0}=\prod_{\alpha \in R^{+}} \prod_{i=1}^{k_{\alpha}-1} \frac{\left[\left(\alpha^{\vee}, \rho_{k}\right)+i\right]}{\left[\left(\alpha^{\vee}, \rho_{k}\right)-i\right]},
$$

which is known as constant term identities. These identities were first conjectured by Macdonald ([M3]), though some special cases had been known before. The classical $(q=1)$ case of these identities was proven by Opdam ([O3]), who used the technique of shift operators, which we will discuss later. In the general case, these identities had been proven case-by-case for most root systems, with the exception of the $E$ series (see [BZ], [GG], [Ha], [K]).

In the case where all $k_{\alpha}$ coincide: $k_{\alpha}=k,(2.7)$ can be rewritten in the following form:

$$
\left[\prod_{\alpha \in R^{+}} \prod_{i=0}^{k-1}\left(1-q^{2 i} e^{\alpha}\right)\left(1-q^{2 i+2} e^{-\alpha}\right)\right]_{0}=\prod\left[\begin{array}{c}
k d_{i} \\
k
\end{array}\right],
$$

where $\left[\begin{array}{l}a \\ b\end{array}\right]=\frac{[a] !}{[b] ![a-b] !}$ is the $q$-binomial coefficient, and $d_{i}$ are the exponents of the Weyl group $W$, or the degrees of the free generators of $(S[V])^{W}$. This reformulation is not trivial: it involves some identities for the Poincaré series of $W$, which can be found in [M4]. In particular, for $A_{n-1}$ the exponents are $2,3, \ldots, n$, and formula $\left(2.7^{\prime}\right)$ becomes

$$
\left[\prod_{i<j}^{k-1}\left(1-q^{2 l} \frac{x_{i}}{x_{j}}\right)\left(1-q^{2 l+2} \frac{x_{j}}{x_{i}}\right)\right]_{0}=\frac{[n k] !}{[k] !^{n}} .
$$

It is also worth noting that if we let $k \rightarrow \infty$ in $\left(2.7^{\prime}\right)$, then the formula we get is closely related with the denominator identity for the corresponding affine root system, which is also due to Macdonald.

\section{LeCture 3: Affine Hecke algebras and induced Representations}

In this lecture we define and study the affine Hecke algebras. Most of the results we give in this lecture are due to Lusztig ([L]); some special cases of these results were first proven by Bernstein and Zelevinsky (unpublished).

We start with the definition of an affine Weyl group. In the notation of the previous sections, let $\widehat{V}=V \oplus \mathbb{R} \delta$; we will interpret elements of $\widehat{V}$ as functions on $V$ by $(v+k \delta)\left(v^{\prime}\right)=\left(v, v^{\prime}\right)+k$. Define the affine root system $\widehat{R}=R \times \mathbb{Z} \delta$ and the positive affine roots $\widehat{R}^{+}=\left\{\alpha+k \delta \mid \alpha \in R, k>0\right.$ or $\left.\alpha \in R^{+}, k \geq 0\right\}$. The basis of simple roots in $\widehat{R}^{+}$is given by $\alpha_{0}=-\theta+\delta, \alpha_{1}, \ldots, \alpha_{n}$. Equivalently, $\widehat{R}^{+}=\{\widehat{\alpha} \in \widehat{R} \mid \widehat{\alpha}$ is non-negative on $C\}$, where $C=\left\{v \in V \mid \alpha_{i}(v) \geq 0, i=0 \ldots n\right\}$ is the positive affine Weyl chamber.

For every $\widehat{\alpha} \in \widehat{R}$ we define the reflection $s_{\widehat{\alpha}}: \widehat{V} \rightarrow \widehat{V}$ by

$$
s_{\widehat{\alpha}}: \widehat{\lambda} \mapsto \widehat{\lambda}-\left(\lambda, \alpha^{\vee}\right) \widehat{\alpha},
$$


where $\widehat{\lambda}=\lambda+m \delta, \widehat{\alpha}=\alpha+k \delta$. Note that this action preserves $\widehat{R}$. The dual action of $s_{\widehat{\alpha}}$ on $V$ is just the reflection of $V$ with respect to the (affine) hyperplane $\widehat{\alpha}(v)=0$ :

$$
s_{\widehat{\alpha}}: v \mapsto v-\widehat{\alpha}(v) \alpha^{\vee} .
$$

We will use the notation $s_{0} \ldots s_{n}$ for $s_{\alpha_{0}} \ldots s_{\alpha_{n}}$. Let the affine Weyl group $W^{a}$ be the group generated by $s_{\widehat{\alpha}}$. Then the following facts are well-known:

Theorem 3.1. 1. $W^{a}=W \ltimes \tau\left(Q^{\vee}\right)$, where the action of $\alpha^{\vee} \in Q^{\vee}$ on $\widehat{V}$ is given by

$$
\tau\left(\alpha^{\vee}\right): \widehat{\lambda} \mapsto \widehat{\lambda}-\left(\alpha^{\vee}, \lambda\right) \delta .
$$

(Respectively, the dual action on $V$ is given by $\tau\left(\alpha^{\vee}\right): v \mapsto v+\alpha^{\vee}$.)

In particular, $s_{0}=\tau\left(\theta^{\vee}\right) s_{\theta}=s_{\theta} \tau\left(-\theta^{\vee}\right)$.

2. $W^{a}$ is generated by $s_{0}, \ldots, s_{n}$ with the relations $s_{i}^{2}=1$ and the Coxeter relations: for $i \neq j$

$$
s_{i} s_{j} s_{i} \ldots=s_{j} s_{i} s_{j} \ldots \quad m_{i j} \text { terms on each side, }
$$

where $m_{i j}$ are defined in the standard way from the corresponding affine Dynkin diagram. (For the root system $\hat{A}_{1}$ there are no Coxeter relations, which is sometimes formally expressed by letting $m_{i j}=\infty$.)

3. For every $w \in W^{a}$, its length $l(w)$ with respect to the generators $s_{0}, \ldots, s_{n}$ is equal to

$$
l(w)=\left|\widehat{R}^{+} \cap w^{-1} \widehat{R}^{-}\right| .
$$

4. Let $w=s_{i_{l}} \ldots s_{i_{1}}$ be a reduced expression. Define the associated sequence of affine roots by

$$
\alpha^{(1)}=\alpha_{i_{1}}, \alpha^{(2)}=s_{i_{1}}\left(\alpha_{i_{2}}\right), \ldots, \alpha^{(l)}=s_{i_{1}} \ldots s_{i_{l-1}}\left(\alpha_{i_{l}}\right) .
$$

Then

$$
\left\{\alpha^{(1)}, \ldots, \alpha^{(l)}\right\}=\widehat{R}^{+} \cap w^{-1} \widehat{R}^{-} .
$$

5. $C$ is a fundamental domain for the action of $W^{a}$ on $V$.

Define the extended affine Weyl group $\widetilde{W}$ as the semidirect product $\widetilde{W}=W \ltimes$ $\tau\left(P^{\vee}\right)$, where the action of $\tau\left(P^{\vee}\right)$ is given by the same formulas as we had before for $\tau\left(Q^{\vee}\right)$. Note that the action of the extended Weyl group on $\widehat{V}$ preserves $\widehat{R}$. It is easy to see that $W^{a}$ is a normal subgroup in $\widetilde{W}$ and that $\widetilde{W} / W^{a} \simeq P^{\vee} / Q^{\vee}$ is a finite abelian group, whose elements are in one-to-one correspondence with the minuscule coweights (see Lemma 2.2).

Let us define the length $l(\tilde{w})$ of an arbitrary $\tilde{w} \in \widetilde{W}$ by formula (3.5). In general, $\widetilde{W}$ is not a Coxeter group, and $l(\tilde{w})$ cannot be interpreted as the length of a reduced decomposition. Moreover, there are elements of length 0. Define

$$
\Omega=\{\tilde{w} \in \widetilde{W} \mid l(\tilde{w})=0\}=\{\tilde{w} \in \widetilde{W} \mid \tilde{w}(C)=C\} .
$$

Obviously, $\Omega$ is a subgroup, and it follows from Theorem 3.1(5) that $\widetilde{W}=\Omega \ltimes W^{a}$; hence, $\Omega \simeq P^{\vee} / Q^{\vee}$. This means that every element of $\Omega$ has the form $\pi_{r}=\tau\left(b_{r}\right) w_{r}$ for some minuscule coweight $b_{r}$ and $w_{r} \in W^{a}$. It is also useful to note that $\pi_{r}$ acts 
on the simple roots $\alpha_{0} \ldots \alpha_{n}$ by some permutation; in particular, $\pi_{r}\left(\alpha_{0}\right)=\alpha_{r}$. Thus, we get the following description of $\widetilde{W}$ :

$$
\widetilde{W}=\Omega \ltimes W^{a},
$$

with the relation $\pi s_{i} \pi^{-1}=s_{j}$ if $\pi\left(\alpha_{i}\right)=\alpha_{j}$.

We will need some properties of the length function $l(\tilde{w})$.

Lemma 3.2. $\quad$ 1. $l(\pi \tilde{w})=l(\tilde{w}), \quad \pi \in \Omega$

2. $l\left(\tilde{w} s_{i}\right)= \begin{cases}l(\tilde{w})+1, & \tilde{w}\left(\alpha_{i}\right) \in \widehat{R}^{+} \\ l(\tilde{w})-1, & \tilde{w}\left(\alpha_{i}\right) \in \widehat{R}^{-}\end{cases}$

3. If $w \in W, \lambda \in P^{\vee}$, then

$$
l(w \tau(\lambda))=\sum_{\alpha \in R^{+}}|(\lambda, \alpha)+\chi(w \alpha)|,
$$

where $\chi(\alpha)=0$ if $\alpha \in R^{+}$and $\chi(\alpha)=1$ if $\alpha \in R^{-}$.

Proof. (1) is obvious from the definition; (2) follows from (1) and the standard results about affine Weyl groups; (3) can be derived straightforwardly from the definition of length.

Corollary 3.3. $\quad$ 1. If $\lambda \in P^{\vee}$, then $l(\tau(\lambda))=2\left(\lambda^{+}, \rho\right)$, where $\lambda^{+}$is the dominant coweight lying in the $W$-orbit of $\lambda$.

2. If $\lambda \in P_{+}^{\vee}$, then $l(w \tau(\lambda))=l(w)+l(\tau(\lambda))$.

3. If $\left(\lambda, \alpha_{i}\right)=0$ for some $i \in\{1, \ldots, n\}$, then $l\left(\tau(\lambda) s_{i}\right)=l\left(s_{i} \tau(\lambda)\right)=l(\tau(\lambda))+1$.

4. If $\left(\lambda, \alpha_{i}\right)=-1$, then $l\left(s_{i} \tau(\lambda)\right)=l(\tau(\lambda))-1$.

Now we can define the braid group.

Definition 3.4. The braid group $B$ is the group generated by the elements $T_{\tilde{w}}, \tilde{w} \in$ $\widetilde{W}$ subject to the following relations:

$$
T_{v} T_{w}=T_{v w} \quad \text { if } l(v w)=l(v)+l(w), \quad v, w \in \widetilde{W} .
$$

In particular, this implies that the elements $T_{\pi}, \pi \in \Omega$ form a subgroup in $B$ which is isomorphic to $\Omega$; abusing the language, we will use the same notation $\pi$ for $T_{\pi}$. Also, we will write $T_{i}$ for $T_{s_{i}}, i=0 \ldots n$.

Lemma 3.5. $T_{\tilde{w} s_{i}}=T_{\tilde{w}} T_{i}$ if $\tilde{w} s_{i}$ is a reduced expression, and $T_{\tilde{w} s_{i}}=T_{\tilde{w}} T_{i}^{-1}$ otherwise.

Proof. Immediately follows from Lemma 3.2(2).

\section{Theorem 3.6.}

$$
B=\Omega \ltimes B\left(T_{0}, T_{1}, \ldots, T_{n}\right),
$$

where $B\left(T_{0}, \ldots, T_{n}\right)$ is the group with generators $T_{0}, \ldots, T_{n}$ and relations (3.4) (Coxeter relations), and the action of $\Omega$ on $T_{i}$ is given by $\pi T_{i} \pi^{-1}=T_{j}$ if $\pi\left(\alpha_{i}\right)=\alpha_{j}$.

Proof. This theorem immediately follows from the previous results and the following well-known result, due to Iwahori and Matsumoto: for any two reduced expressions for an element $\tilde{w} \in W^{a}$ one can be obtained from another by a sequence of Coxeter relations (3.4) without using the relations $s_{i}^{2}=1$.

Now we can do one of the most crucial steps. Define elements $Y^{\lambda} \in B$ for $\lambda \in P^{\vee}$ thus: 
1. If $\lambda \in P_{+}^{\vee}$, then $Y^{\lambda}=T_{\tau(\lambda)}$.

2. If $\lambda=\mu-\nu$, with $\mu, \nu \in P_{+}^{\vee}$, then $Y^{\lambda}=Y^{\mu}\left(Y^{\nu}\right)^{-1}$.

Theorem 3.7. $\quad 1$. $Y^{\lambda}$ is well-defined for all $\lambda$, and $Y^{\lambda} Y^{\mu}=Y^{\lambda+\mu}$.

2. Let $\tau(\lambda)=\pi_{r} s_{i_{l}} \ldots s_{i_{1}}$ be a reduced expression, and let $\alpha^{(1)}, \ldots, \alpha^{(l)}$ be the associated sequence of affine roots (see (3.6)). Then $Y^{\lambda}=\pi_{r} T_{i_{l}}^{\varepsilon_{l}} \ldots T_{i_{1}}^{\varepsilon_{1}}$, where $\varepsilon_{i}=1$ if the corresponding $\alpha^{(i)}$ has the form $\alpha^{(i)}=\alpha+k \delta, \alpha \in R^{+}$, and $\varepsilon_{i}=-1$ otherwise. In particular, if $\lambda \in P_{+}^{\vee}$, then all $\varepsilon_{i}=1$; and if $\lambda \in P_{-}^{\vee}$, then all $\varepsilon_{i}=-1$.

Proof. (1) It follows from Corollary 3.3(1) that $T_{\tau(\lambda+\mu)}=T_{\tau(\lambda)} T_{\tau(\mu)}$ if $\lambda, \mu \in P_{+}^{\vee}$. This implies (1).

As for (2), the proof consists of several steps which we briefly outline. Let us call an affine root $\widehat{\alpha} R$-positive if $\widehat{\alpha}=\alpha+k \delta, \alpha \in R^{+}$.

Lemma. For any (not necessarily reduced) expression $\tau(\lambda)=\pi_{r} s_{i_{l}} \ldots s_{i_{1}}$ denote $\tilde{Y}^{\lambda}=\pi_{r} T_{i_{l}}^{\varepsilon_{l}} \ldots T_{i_{1}}^{\varepsilon_{1}}$, where the signs $\varepsilon_{i}$ are determined as in the statement of the theorem. Then $\tilde{Y}^{\lambda}$ does not depend on the choice of the expression for $\tau(\lambda)$.

Proof. First, show that if $\tau(\lambda)=x s_{i}^{2} y=x y, x, y \in \widetilde{W}$ are two expressions for $\tau(\lambda)$, then the corresponding expressions for $\tilde{Y}^{\lambda}$ are equal. Indeed, the sequence of roots associated with the first expression differs from the second one by insertion of the pair $\alpha^{(k)}=y^{-1} \alpha_{i}, \alpha^{(k+1)}=y^{-1}\left(-\alpha_{i}\right)$. Since precisely one of these roots is $R$-positive, we have $T_{i}^{\varepsilon_{k+1}} T_{i}^{\varepsilon_{k}}=1$.

Similarly, if $\tau(\lambda)=x\left(s_{i} s_{j}\right)^{m_{i j}} y=x y, x, y \in \widetilde{W}$, where $m_{i j}$ is as in (3.4), are two expressions for $\tau(\lambda)$, then the sequence of roots associated with the first expression differs from the second one by insertion of the set of roots $\left\{y^{-1} \beta_{k}\right\}_{\beta_{k} \in R<\alpha_{i}, \alpha_{j}>\text {, }}$ where $R<\alpha_{i}, \alpha_{j}>$ is the root system of rank two spanned by $\alpha_{i}, \alpha_{j}$. Moreover, these roots appear in their natural cyclic order: one can choose an orientation in $\mathbb{R} \alpha_{i} \oplus \mathbb{R} \alpha_{j}$ such that the roots $\beta_{k}$ appear in the counterclockwise order. Both of these facts can be easily checked case-by-case, since one has to consider only root systems of rank 2 . Since the condition of being an $R$-positive root specifies a halfspace, we see that among the roots $y^{-1} \beta_{k}$ precisely half (i.e. $m_{i j}$ ) are $R$-positive, and they are grouped together. Thus, the corresponding part of the expression for $\tilde{Y}^{\lambda}$ has the form

$$
\underbrace{T_{i} T_{j} T_{i} \ldots}_{p \text { "+" signs }} \underbrace{\ldots T_{i}^{-1} T_{j}^{-1} \cdots}_{m_{i j} \text { "-" signs }} \underbrace{\ldots T_{i} T_{j}}_{m_{i j}-p \text { "+" signs }}
$$

or

$$
\underbrace{T_{i}^{-1} T_{j}^{-1} T_{i}^{-1} \cdots}_{p \text { "-" signs }} \underbrace{\ldots T_{i} T_{j} \ldots}_{m_{i j} \text { "+" signs }} \underbrace{\ldots T_{i}^{-1} T_{j}^{-1}}_{m_{i j}-p \text { "-" signs }}
$$

for some $p$. In both cases, this product is equal to 1, which completes the proof of the lemma.

Now it is relatively easy to prove (2). Indeed, let $\lambda \in P_{+}^{\vee}$, and let $\tau(\lambda)=$ $\pi_{r} s_{i_{l}} \ldots s_{i_{1}}$ be a reduced expression. Then $\left\{\alpha^{(1)}, \ldots, \alpha^{(l)}\right\}=\left\{\widehat{\alpha} \in \widehat{R}^{+} \mid \tau(\lambda) \widehat{\alpha} \in\right.$ $\left.\widehat{R}^{-}\right\}$, and explicit calculation shows that all of them are $R$-positive, and therefore $\tilde{Y}^{\lambda}=\pi_{r} T_{i_{l}} \ldots T_{i_{1}}=Y^{\lambda}$. The previous lemma and the fact that $\widehat{\alpha}$ is $R$-positive iff 
$\tau(\lambda) \widehat{\alpha}$ is $R$-positive imply that $\tilde{Y}^{\lambda} \tilde{Y}^{\mu}=\tilde{Y}^{\lambda+\mu}$. Thus, $\tilde{Y}^{\lambda}=Y^{\lambda}$ for all $\lambda \in P^{\vee}$, which concludes the proof of $(2)$.

Thus, the subgroup generated by $Y^{\lambda}$ is isomorphic to the coweight lattice $P^{\vee}$.

Lemma 3.8. 1. The elements $Y^{\lambda}, \lambda \in P^{\vee}, T_{1}, \ldots, T_{n}$ generate $B$ as a group.

2. If $\left(\lambda, \alpha_{i}\right)=0$ for some $i, 1 \leq i \leq n$, then $T_{i} Y^{\lambda}=Y^{\lambda} T_{i}$.

3. If $\left(\lambda, \alpha_{i}\right)=1$ for some $i, 1 \leq i \leq n$, then $Y^{\lambda}=T_{i} Y^{s_{i} \lambda} T_{i}$. (Note: This is not a misprint: there are two $T_{i}$ 's and no $T_{i}^{-1}$ in the formula.)

Proof. 1. Since every $\tilde{w} \in \widetilde{W}$ can be written as $\tilde{w}=\tau\left(b_{r}\right) w$ for some minuscule (and thus, dominant) weight $b_{r}$ and $w \in W^{a}$, it follows from Lemma 3.5 that $Y^{\lambda}, T_{0}, T_{1}, \ldots T_{n}$ generate $B$. Similarly, it follows from $s_{0}=\tau\left(\theta^{\vee}\right) s_{\theta}$ that $T_{0}$ can be written in terms of $Y^{\theta^{\vee}}$ and $T_{1}, \ldots, T_{n}$.

2. If $\lambda \in P_{+}^{\vee}$, then (2) follows immediately from Corollary 3.3(3). For general $\lambda$ this statement follows from the fact that every $\lambda$ can be written in the form $\lambda=\mu-\nu$ with $\mu, \nu \in P_{+}^{\vee},\left(\mu, \alpha_{i}\right)=\left(\nu, \alpha_{i}\right)=0$.

3. Suppose $\lambda \in P_{+}^{\vee}$. Introduce $\pi=\lambda+s_{i}(\lambda)=2 \lambda-\alpha_{i}^{\vee}$; then $\pi \in P_{+}^{\vee}$. If $l(\tau(\lambda))=2(\lambda, \rho)=p$, then $l(\tau(\pi))=2 p-2$, and it follows from Lemma 3.2(2) that $l\left(\tau(\lambda) s_{i}\right)=p-1$. Thus, if we write $s_{i} \tau(\pi)=\left(\tau(\lambda) s_{i}\right)(\tau(\lambda))$ (it is easy to see that it is in fact an identity in $\widetilde{W})$, then both left-hand and righthand sides are reduced expressions, and thus $T_{i} Y^{\pi}=T_{\tau(\lambda) s_{i}} Y^{\lambda}=Y^{\lambda} T_{i}^{-1} Y^{\lambda}$, which is equivalent to the desired equality.

If $\lambda \notin P_{+}^{\vee}$, then it can be written as $\lambda=\mu-\nu$, with $\mu, \nu \in P_{+}^{\vee},\left(\mu, \alpha_{i}\right)=$ $1,\left(\nu, \alpha_{i}\right)=0$, and thus the statement follows from the previous arguments.

Now we can give a definition of an affine Hecke algebra. Suppose that for every $\alpha \in \widehat{R}$ we have a variable $t_{\alpha}$ such that $t_{\alpha}=t_{w(\alpha)}$ for every $w \in \widetilde{W}$ (thus, there are at most two different $t^{\prime}$ s). Let $\mathbb{C}_{t}=\mathbb{C}\left(t_{\alpha}\right)$ be the field of rational functions in $t_{\alpha}$.

Definition. The affine Hecke algebra $\widehat{H}$ is the quotient of the group algebra $\mathbb{C}_{t}[B]$ by the ideal generated by the following relations:

$$
\left(T_{i}-t_{i}\right)\left(T_{i}+t_{i}^{-1}\right)=0, \quad i=0, \ldots, n,
$$

where $t_{i}=t_{\alpha_{i}}$ (in particular, $t_{0}=t_{\alpha_{0}}=t_{\theta}$ ).

Note that these relations imply that $T_{i}^{-1}=T_{i}+\left(t_{i}^{-1}-t_{i}\right)$.

In a similar way, we can define $H^{a}$ as a subalgebra of $\widehat{H}$ generated by $T_{0}, \ldots, T_{n}$ and the non-affine Hecke algebra $H$ as a subalgebra generated by $T_{1}, \ldots, T_{n}$, so $H \subset H^{a} \subset \widehat{H}$ (which is a complete analogue of $W \subset W^{a} \subset \widetilde{W}$ ). We will also use the notation $\mathbb{C}_{t}[Y]$ for the commutative subalgebra in $\widehat{H}$ generated by $Y^{\lambda}$.

Theorem 3.9. $\widehat{H}=\Omega \ltimes H^{a}$, where the action of $\Omega$ on $T_{i}$ is the same as in Theorem 3.6 .

Lemma 3.10. One has the following relations in $\widehat{H}$ :

$$
T_{i} Y^{\lambda}-Y^{s_{i}(\lambda)} T_{i}=\left(t_{i}-t_{i}^{-1}\right) \frac{Y^{s_{i}(\lambda)}-Y^{\lambda}}{Y^{-\alpha_{i}^{\vee}}-1}, \quad i=1, \ldots, n
$$

(from now on, expressions of the form $\frac{A}{B}$ stand for $B^{-1} A$, though in this case the order is not important). 
Remark. It is easy to see that the right-hand side is in fact a polynomial, i.e. lies in $\mathbb{C}_{t}[Y]$.

Proof. First, simple calculation shows that if this relation is true for $Y^{\lambda}$ and $Y^{\mu}$, then it is also true for $Y^{\lambda+\mu}$ and $Y^{-\lambda}$. Thus, it suffices to prove (3.10) when $\left(\lambda, \alpha_{i}\right)=0$ or $\left(\lambda, \alpha_{i}\right)=1$. If $\left(\lambda, \alpha_{i}\right)=0,(3.10)$ reduces to $T_{i} Y^{\lambda}=Y^{\lambda} T_{i}$, which is Lemma 3.8(2). Similarly, if $\left(\lambda, \alpha_{i}\right)=1$, then $Y^{s_{i}(\lambda)}=Y^{\lambda-\alpha_{i}^{\vee}}$, and (3.10) reduces to $T_{i} Y^{\lambda}-Y^{s_{i}(\lambda)} T_{i}=\left(t_{i}-t_{i}^{-1}\right) Y^{\lambda}$, which is an immediate corollary of Lemma 3.8 and the identity $T_{i}=T_{i}^{-1}+\left(t_{i}-t_{i}^{-1}\right)$.

Theorem 3.11. $\quad$ 1. $\widehat{H}=H \cdot \mathbb{C}_{t}[Y]$, where the commutation relations of $H$ and $Y$ are given by $(3.10)$.

2. Every element of $\widehat{H}$ can be uniquely written in either of the following forms:

$$
h=\sum_{w \in W} p_{w}(Y) T_{w}=\sum_{w \in W} T_{w} q_{w}(Y) .
$$

3. The subalgebra in $\widehat{H}$ generated by $Y^{\lambda}$ is isomorphic to the group algebra of the coweight lattice: $\mathbb{C}_{t}[Y] \simeq \mathbb{C}_{t}\left[P^{\vee}\right]$.

Proof. One direction is rather easy. First, it follows from Lemma 3.8 that the elements $T_{w}, w \in W$ and $Y^{\lambda}, \lambda \in P^{\vee}$ generate $\widehat{H}$ as an algebra. Now relations (3.10) imply that every element $h$ can be written in the form $h=\sum_{w} T_{w} q_{w}(Y)$, which proves the existence part of the theorem.

To prove uniqueness, we must show that these elements are linearly independent. This is more difficult, and we do not give the proof here, referring the reader to $[\mathrm{L}$, Lemma 3.4].

Theorem 3.12. The center of $\widehat{H}$ coincides with the subspace of $W$-invariants in $\mathbb{C}_{t}[Y] \subset \widehat{H}: \mathcal{Z}(\widehat{H})=\mathbb{C}_{t}[Y]^{W}$.

Proof. It is easy to check, using (3.10), that $\mathbb{C}_{t}[Y]^{W} \subset \mathcal{Z}(\widehat{H})$. On the other hand, in the specialization $t_{i}=1$ we have $\mathcal{Z}(\widehat{H})=\mathbb{C}[Y]^{W}$, which is easy to prove; thus, the same must be true for generic $t_{i}$.

We will also need some information about the representations of $\widehat{H}$. The general theory of representations of $\widehat{H}$ is rather complicated (see [L]); however, we will use only representations of some special form. Namely, let $E$ be an arbitrary representation of $H$; it is known that for generic values of $t_{i}$ the finite-dimensional Hecke algebra $H$ is isomorphic to the group algebra $\mathbb{C}_{t}[W]$, and thus has the same representations. Define a representation $\widehat{E}$ of $\widehat{H}$ as the induced representation: $\widehat{E}=$ $\operatorname{Ind}_{H}^{\widehat{H}} E$. It follows from Theorem 3.11 that as a vector space, $\widehat{E}=\mathbb{C}_{t}[Y] \otimes E$, and $\mathbb{C}_{t}[Y]$ acts by left multiplication. In particular, let us take the trivial representation of $H$; that is, let $E=\mathbb{C}_{t}, T_{i} \mapsto t_{i}, i=1, \ldots, n$. Then we get an action of $\widehat{H}$ on the space $\mathbb{C}_{t}[Y]$.

Theorem 3.13. The above defined action of $\widehat{H}$ on $\mathbb{C}_{t}[Y]$ is given by

$$
\begin{gathered}
Y^{\lambda} \mapsto Y^{\lambda} \\
T_{i} \mapsto t_{i} s_{i}+\left(t_{i}-t_{i}^{-1}\right) \frac{s_{i}-1}{Y^{-\alpha_{i}^{\vee}-1}}, \quad i=1, \ldots, n
\end{gathered}
$$

where $s_{i}$ stands for the usual action of $W$ on $\mathbb{C}_{t}[Y]: s_{i} Y^{\lambda}=Y^{s_{i}(\lambda)}$. 
Proof. Immediately follows from Lemma 3.10.

Proposition 3.14. The above defined representation of $\widehat{H}$ is faithful.

Remark. The degenerate affine Hecke algebra which we defined in Lecture 1 (for the root system $A_{n-1}$ ) can be obtained as the following degeneration of the affine Hecke algebra $\widehat{H}$ : write (formally) $Y^{\lambda}=t^{y_{\lambda} / h}$. Then as $t \rightarrow 1$ the relations for $y_{\lambda}, T_{i}$ become the relations of the degenerate affine Hecke algebra.

\section{Lecture 4: Double affine Hecke algebras and Commuting difference OPERATORS}

In this lecture, we will start applying the results about the affine Hecke algebras, which we discussed before, to the Macdonald's theory. Unless otherwise stated, all constructions and results in this lecture are due to Cherednik.

Recall that for any root system we have defined the affine Hecke algebra $\widehat{H}$ which has two descriptions:

1. $\widehat{H}$ is generated by elements $T_{0}, \ldots, T_{n}$ and by $\pi \in \Omega$ with relations

$$
\begin{gathered}
\text { Coxeter relations for } T_{i}, i=0, \ldots, n \\
\begin{array}{c}
\left(T_{i}-t_{i}\right)\left(T_{i}+t_{i}^{-1}\right)=0, i=0, \ldots, n \\
\pi T_{i} \pi^{-1}=T_{j} \text { if } \pi\left(\alpha_{i}\right)=\alpha_{j} .
\end{array}
\end{gathered}
$$

2. $\widehat{H}=H \cdot \mathbb{C}_{t}[Y]$, where $H$ is the Hecke algebra generated by $T_{1}, \ldots, T_{n}$ with the relations above, $\mathbb{C}_{t}[Y]$ is the algebra generated by $Y^{\lambda}, \lambda \in P^{\vee}$ such that $Y^{\lambda+\mu}=Y^{\lambda} Y^{\mu}$ (thus, $\mathbb{C}_{t}[Y] \simeq \mathbb{C}_{t}\left[P^{\vee}\right]$ ), and the commutation relations between $T_{i}, Y^{\lambda}$ are

$$
\begin{aligned}
T_{i} Y^{\lambda}=Y^{\lambda} T_{i} & \text { if }\left(\lambda, \alpha_{i}\right)=0 \\
T_{i} Y^{\lambda}-Y^{s_{i}(\lambda)} T_{i}=\left(t_{i}-t_{i}^{-1}\right) Y^{\lambda} & \text { if }\left(\lambda, \alpha_{i}\right)=1 .
\end{aligned}
$$

Our goal is to use this algebra (along with its action on $\mathbb{C}_{t}[Y]$ defined in Theorem 3.13) to construct a commuting family of difference operators, similar to the construction in Lecture 1 where the degenerate affine Hecke algebra was used to construct a commuting family of differential operators.

Note, however, that in Lecture 1 both the operators $\partial_{i}=\frac{\partial}{\partial x_{i}}$ and the degenerate affine Hecke algebra were used to construct commuting differential operators; one can say that we have used the algebra generated by $\partial_{i}, x_{i}$ and $\hat{s}_{i}$. It turns out that in order to construct a commuting family of difference operators, one needs a $q$-analogue of this larger algebra. Such an analogue was constructed by Cherednik, who called it "the double affine Hecke algebra". This is an algebra generated by three sets of variables:

1. $T_{i}, i=1 \ldots n$

2. $Y^{\lambda}, \lambda \in P^{\vee}$

3. $X^{\mu}, \mu \in P$

Here $Y^{\lambda}, T_{i}$ satisfy the relations of the affine Hecke algebra above. Since $W$ is the Weyl group for $R^{\vee}$ as well as for $R$, we can define the relations between $X^{\mu}$ and $T_{i}$ to be the relations of the affine Hecke algebra for the root system $R^{\vee}$, i.e. the same relations as above with $Y^{\lambda}$ replaced by $X^{\mu}, \mu \in P$ and $\alpha_{i}$ replaced by $\alpha_{i}^{\vee}$.

However, in this realization it is rather difficult to describe the relations between $Y^{\lambda}, X^{\mu}$. For this reason, let us replace the generators $Y^{\lambda}, T_{i}$ with $\pi \in \Omega, T_{0}, \ldots, T_{n}$ 
which generate the same affine Hecke algebra $\widehat{H}^{Y}$. Thus, instead of describing the relations between $Y^{\lambda}$ and $X^{\mu}$ we will write the relations between $T_{0}, \pi$ and $X^{\mu}$.

Let us consider the affine weight lattice $\widehat{P}=\left\{\mu+k \delta \mid \mu \in P, k \in \frac{1}{m} \mathbb{Z}\right\} \subset \hat{V}$, where $m \in \mathbb{Z}_{+}$is the smallest integer such that $m(\lambda, \mu) \in \mathbb{Z}$ for every $\lambda \in P^{\vee}, \mu \in P$. Then we have a natural action of the extended affine Weyl group $\widetilde{W}=W \ltimes \tau\left(P^{\vee}\right)$ on $\widehat{P}$ and thus on the group algebra $\mathbb{C}[\widehat{P}]$ spanned by $X^{\hat{\mu}}$. Let $q$ be an independent variable, and $\mathbb{C}_{q}$ the field of rational functions in $q^{1 / m}$. Define the embedding $\mathbb{C}[\widehat{P}] \subset \mathbb{C}_{q}[P]$ by $X^{\delta}=q^{-2}$. The action of $\widetilde{W}$ on $\mathbb{C}[\widehat{P}]$ can be extended by $\mathbb{C}_{q^{-}}$ linearity to $\mathbb{C}_{q}[P]$; in particular,

$$
\begin{gathered}
\tau(\lambda) X^{\mu}=q^{2(\lambda, \mu)} X^{\mu} \\
s_{0} X^{\mu}=X^{\mu}\left(X^{\theta} q^{2}\right)^{-\left(\mu, \theta^{\vee}\right)} .
\end{gathered}
$$

Definition 4.1. Let $\mathbb{C}_{q, t}$ be the field of rational functions in $q, t_{\alpha}$. Then the double affine Hecke algebra $\mathcal{H}$ is an algebra over $\mathbb{C}_{q, t}$ which is generated by the elements $\pi \in \Omega, T_{0}, \ldots, T_{n}, X^{\mu}, \mu \in P$ subject to the following relations:

1. The relations (a)-(c) of the affine Hecke algebra between $T_{i}, \pi_{r}$.

2. $X^{\mu+\nu}=X^{\mu} X^{\nu}, X^{0}=1$.

3

$$
\begin{gathered}
T_{i} X^{\mu}=X^{\mu} T_{i} \quad \text { if }\left(\mu, \alpha_{i}^{\vee}\right)=0 \\
T_{i} X^{\mu}-X^{s_{i}(\mu)} T_{i}=\left(t_{i}-t_{i}^{-1}\right) X^{\mu} \quad \text { if }\left(\mu, \alpha_{i}^{\vee}\right)=1 .
\end{gathered}
$$

Here $i=0 \ldots n$, and $\alpha_{0}^{\vee}=-\theta^{\vee}$.

4. $\pi X^{\mu} \pi^{-1}=X^{\pi(\mu)}$.

Note that relation (4.2) for $i=0$ reads $T_{0} X^{\mu}-X^{\mu+\theta} q^{2} T_{0}=\left(t_{0}-t_{0}^{-1}\right) X^{\mu}$ if $\left(\mu, \theta^{\vee}\right)=-1$.

Obviously, the subalgebra generated by $\pi, T_{0} \ldots T_{n}$ satisfies the relations of the affine Hecke algebra $\widehat{H}$ defined in the previous lecture (later we will prove that this subalgebra is in fact isomorphic to $\widehat{H}$ ). Thus, we can define the elements $Y^{\lambda}, \lambda \in P^{\vee}$ in $\mathcal{H}$. Similarly, the subalgebra generated by $T_{1}, \ldots, T_{n}, X^{\mu}$ satisfies the relations of affine Hecke algebra for the root system $R^{\vee}$. We will denote these affine Hecke subalgebras by $\widehat{H}^{Y}$ and $\widehat{H}^{X}$ respectively. In fact, in the above definition $X^{\mu}$ and $Y^{\lambda}$ play a symmetric role: $\mathcal{H}$ could be just as well defined as the algebra generated by $Y^{\lambda}, T_{0}^{\prime}, T_{1}, \ldots, T_{n}, \pi^{\prime} \in \Omega^{\vee}$ (see details in [C7]). It is also worth noting that it is rather difficult to write down explicitly the commutation relations between $X^{\mu}$ and $Y^{\lambda}$.

For the root system $A_{n-1}$ the algebra $\mathcal{H}$ can be described topologically: in this case $\mathcal{H}$ is a deformation of the algebra $\mathbb{C}\left[B T_{n}\right] /\left(T_{i}-t_{i}\right)\left(T_{i}+t_{i}^{-1}\right)=0$, where $B T_{n}$ is the braid group of $n$ points on a torus. Under this isomorphism, $X_{i}$ corresponds to the $i$-th point going around the $x$-cycle on the torus, $Y_{i}$ corresponds to the $i$-th point going around the $y$-cycle on the torus, and $T_{i}$ corresponds to the transposition of the $i$-th and $i+1$-th points (see [C1, Definition 4.1]).

Theorem 4.2. Every element $h \in \mathcal{H}$ can be uniquely written in the form

$$
\sum_{\lambda, \mu, w} a_{\lambda \mu w} X^{\mu} Y^{\lambda} T_{w}, \quad a_{\lambda \mu w} \in \mathbb{C}_{q, t} .
$$


Proof. The existence of such a representation is a standard exercise; the uniqueness is highly non-trivial, and we postpone the proof until the next lecture (see Corollary 5.8).

Theorem 4.3. The following formulas give a representation of $\mathcal{H}$ in $\mathbb{C}_{q, t}[X]$ :

$$
\begin{gathered}
X^{\mu} \mapsto X^{\mu} \\
\pi: X^{\mu} \mapsto X^{\pi(\mu)}, \quad \pi \in \Omega \\
T_{i} \mapsto t_{i} s_{i}+\left(t_{i}-t_{i}^{-1}\right) \frac{s_{i}-1}{X^{-\alpha_{i}}-1}, \quad i=0, \ldots, n .
\end{gathered}
$$

Proof. This theorem follows from the analogous statement for affine Hecke algebras (Theorem 3.13). Indeed, all the identities we have to check involve at most two $T_{i}$ 's, and every pair of vertices in an affine Dynkin diagram belongs to some subdiagram of finite type (with the exception of the root system $\hat{A}_{1}$ ). The case of $\hat{A}_{1}$ can be easily checked by direct calculation.

Example. In this representation, $T_{0}$ acts as follows:

$$
T_{0}: X^{\mu} \mapsto\left(t_{0}\left(X^{\theta} q^{2}\right)^{-\left(\mu, \theta^{\vee}\right)}+\left(t_{0}-t_{0}^{-1}\right) \frac{\left(X^{\theta} q^{2}\right)^{-\left(\mu, \theta^{\vee}\right)}-1}{X^{\theta} q^{2}-1}\right) X^{\mu}
$$

It turns out that this representation is faithful (we will prove it later). We will identify elements of $\mathcal{H}$ with the corresponding operators on $\mathbb{C}_{q, t}[X]$.

Let $f \in \mathbb{C}[Y]^{W}=\mathcal{Z}\left(\widehat{H}^{Y}\right)$.

Lemma 4.4. The operators $f \in \mathbb{C}[Y]^{W}=\mathcal{Z}\left(\widehat{H}^{Y}\right) \subset \mathcal{H}$ preserve the subspace $\mathbb{C}_{q, t}[X]^{W} \in \mathbb{C}_{q, t}[X]$.

Proof. Note first that it immediately follows from the formulas in Theorem 4.3 that $p \in \mathbb{C}_{q, t}[X]$ is $W$-invariant if and only if $T_{i} p=t_{i} p$ for all $i=1, \ldots, n$. Now, let $p \in \mathbb{C}_{q, t}[X]^{W}$. Then $T_{i} f p=f T_{i} p=t_{i} f p$ (since $\mathbb{C}[Y]^{W}$ is the center of $\widehat{H}^{Y}$ ), and thus $f p \in \mathbb{C}_{q, t}[X]^{W}$.

It is clear from Theorem 4.3 that for every $h \in \mathcal{H}$ the corresponding operator on $\mathbb{C}_{q, t}[X]$ can be written in the form

$$
h=\sum_{\substack{\lambda \in P^{\vee} \\ w \in W}} g_{\lambda, w} \tau(\lambda) w
$$

for some $g_{\lambda, w} \in \mathbb{C}_{q, t}[X]\left(X^{\alpha}-1\right)^{-1}$ (recall that $\tau(\lambda)$ acts on $\mathbb{C}_{q, t}[X]$ by $X^{\mu} \mapsto$ $\left.q^{2(\lambda, \mu)} X^{\mu}\right)$. In particular, the same is true for $Y^{\lambda} \in \widehat{H}^{Y} \subset \mathcal{H}$. For every operator of the form (4.4) define its restriction by

$$
\operatorname{Res}\left(\sum g_{\lambda, w} \tau(\lambda) w\right)=\sum g_{\lambda, w} \tau(\lambda) .
$$

This definition is chosen so that (1) Res $D$ is a difference operator (that is, it only involves the operators $\tau(\lambda)$ and rational functions in $X$, not the action of $W$ ) and (2) if $D$ preserves $\mathbb{C}_{q, t}[X]^{W}$, then so does $\operatorname{Res} D$, and $\left.(\operatorname{Res} D)\right|_{\mathbb{C}_{q, t}[X]^{W}}=\left.D\right|_{\mathbb{C}_{q, t}[X]^{W}}$.

Then Lemma 4.4 immediately implies:

Theorem 4.5. The operators $L_{f}=\operatorname{Res} f, f \in \mathbb{C}_{q, t}[Y]^{W}$ commute and are $W$ invariant. 
Proof. First, $\left.L_{f}\right|_{\mathbb{C}_{q, t}[X]^{W}}=\left.f\right|_{\mathbb{C}_{q, t}[X]^{W}}$. Thus, $L_{f} L_{g} p=L_{g} L_{f} p$ for every symmetric polynomial $p$. Since both $L_{f}, L_{g}$ are difference operators (they do not contain the action of the Weyl group), it is a well-known fact that this implies $L_{f} L_{g}=$ $L_{g} L_{f}$.

Thus, we have constructed a commutative family of $W$-invariant difference operators on $\mathbb{C}_{q, t}[X]$, labeled by $f \in \mathbb{C}_{q, t}[Y]^{W}$. In the subsequent lectures we will discuss the relation of these operators with the theory of Macdonald polynomials. In particular, we will show that this family includes the Macdonald's difference operators $D_{\pi}$, constructed in Lecture 2 for minuscule coweights, and that the eigenfunctions of $L_{f}$ are Macdonald polynomials.

Example. Let $R$ be of type $A_{1}$. In this case there is only one positive root $\alpha$, and only one minuscule (co)weight $\rho=\alpha / 2$. The reduced expression for $\tau(\rho)$ is $\tau(\rho)=\pi_{\rho} s_{1}$, where $\pi_{\rho}=\tau(\rho) s_{1}$ is the element of zero length; it acts on simple affine roots by permuting $\alpha_{1}=\alpha$ and $\alpha_{0}=-\alpha+\delta$. Therefore, $Y^{\rho}=T_{\tau(\rho)}=$ $\pi_{\rho} T_{1}, Y^{-\rho}=T_{1}^{-1} \pi_{\rho}=\pi_{\rho} T_{0}^{-1}=\pi_{\rho}\left(T_{0}+\left(t^{-1}-t\right)\right)$.

In this case $\mathbb{C}_{q, t}[X]$ is just the space of polynomials in $X^{ \pm 1 / 2}$, where $X=X^{\alpha}$, and the action of the extended Weyl group is given by

$$
\begin{gathered}
\tau(\rho) X=q^{2} X \\
\pi_{\rho} X=q^{-2} X^{-1},
\end{gathered}
$$

so the action of the corresponding affine Hecke algebra is given by

$$
\begin{aligned}
Y^{\rho} & =\pi_{\rho}\left(t s_{1}+\left(t-t^{-1}\right) \frac{s_{1}-1}{X^{-1}-1}\right)=\tau(\rho)\left(t+\left(t-t^{-1}\right) \frac{1-s_{1}}{X-1}\right), \\
Y^{-\rho} & =\pi_{\rho}\left(t s_{0}+\left(t-t^{-1}\right) \frac{s_{0}-1}{q^{2} X-1}+\left(t^{-1}-t\right)\right) \\
& =\tau(\rho) s_{1}\left(t \tau(\alpha) s_{1}+\left(t-t^{-1}\right) \frac{\tau(\alpha) s_{1}-q^{2} X}{q^{2} X-1}\right) \\
& =t \tau(-\rho)+\left(t-t^{-1}\right) \tau(\rho) \frac{\tau(-\alpha)-q^{2} X^{-1} s_{1}}{q^{2} X^{-1}-1} \\
& =t \tau(-\rho)+\left(t-t^{-1}\right) \frac{\tau(-\rho)-X^{-1} \tau(\rho) s_{1}}{X^{-1}-1} .
\end{aligned}
$$

Thus,

$$
\begin{gathered}
\operatorname{Res} Y^{\rho}=t \tau(\rho) \\
\operatorname{Res} Y^{-\rho}=\frac{t X^{-1}-t^{-1}}{X^{-1}-1} \tau(-\rho)+\left(t-t^{-1}\right) \frac{1}{X-1} \tau(\rho),
\end{gathered}
$$

so

$$
\operatorname{Res}\left(Y^{\rho}+Y^{-\rho}\right)=\frac{t X-t^{-1}}{X-1} \tau(\rho)+\frac{t X^{-1}-t^{-1}}{X^{-1}-1} \tau(-\rho)
$$

which is nothing but the Macdonald's difference operator $D_{1}$ for the root system $A_{1}$ (cf. formula (2.5)), multiplied by $t^{-1}$. 


\section{LeCTURE 5: MACDONALD'S DIFFERENCE OPERATORS FROM DOUBLE AFFine HECKE ALGEBRAS}

Let us recall some results from the last lecture. We have defined the double affine Hecke algebra $\mathcal{H}$ which is generated by the elements $T_{w}, w \in W, Y^{\lambda}, \lambda \in$ $P^{\vee}, X^{\mu}, \mu \in P$. Also, we have defined its action on the space $\mathbb{C}_{q, t}[X]$, where $X^{\mu}$ acts by multiplication, and $T_{i}, i=0 \ldots n$ act by

$$
T_{i} \mapsto t_{i} s_{i}+\left(t_{i}-t_{i}^{-1}\right) \frac{s_{i}-1}{X^{-\alpha_{i}}-1} .
$$

This allowed us to construct a large family of commuting difference operators on $\mathbb{C}_{q, t}[X]^{W}$.

In this lecture we will establish a connection between this construction and Macdonald's theory; the variables $q, t_{\alpha}$ used in the definition of the double affine Hecke algebra will be identified with the parameters $q, t_{\alpha}$ of Macdonald polynomials. The main result of this lecture is Theorem 5.9, which shows that the Macdonald's operators $D_{\pi}$ introduced in Lecture 2 are special cases of the operators $L_{f}$ obtained from the double affine Hecke algebra.

All the results of this lecture are due to Cherednik ([C7]).

Let us rewrite the action of $T_{i}$ on $\mathbb{C}_{q, t}[X]$ as follows:

$$
T_{i}=s_{i} G\left(\alpha_{i}\right)
$$

where

$$
\begin{aligned}
G(\alpha) & =t_{\alpha}+\left(t_{\alpha}-t_{\alpha}^{-1}\right) \frac{1-s_{\alpha}}{X^{\alpha}-1} \\
& =\frac{t_{\alpha} X^{\alpha}-t_{\alpha}^{-1}}{X^{\alpha}-1}-\left(t_{\alpha}-t_{\alpha}^{-1}\right) \frac{s_{\alpha}}{X^{\alpha}-1}
\end{aligned}
$$

Then $G(\alpha)$ satisfy

$$
\tilde{w} G(\alpha) \tilde{w}^{-1}=G(\tilde{w}(\alpha)), \quad \alpha \in \widehat{R}, \tilde{w} \in \widetilde{W} .
$$

Using this, we can rewrite for arbitrary $\tilde{w} \in \widetilde{W}$ the action of $T_{\tilde{w}}$ as follows

$$
T_{\tilde{w}}=\tilde{w} G\left(\alpha^{(l)}\right) \ldots G\left(\alpha^{(1)}\right),
$$

where $\alpha^{(i)}$ are defined from a reduced expression for $\tilde{w}$ as in (3.6). Recall that

$$
\left\{\alpha^{(1)}, \ldots, \alpha^{(l)}\right\}=R_{\tilde{w}}:=\widehat{R}^{+} \cap \tilde{w}^{-1} \widehat{R}^{-} .
$$

We will also need the expressions for $Y^{\lambda}$. Recall (see Theorem 3.7(2)) that if $\tau(\lambda)=\pi s_{i_{l}} \ldots s_{i_{1}}$ is a reduced expression, then $Y^{\lambda}=\pi T_{i_{l}}^{\varepsilon_{l}} \ldots T_{i_{1}}^{\varepsilon_{1}}$ for some choice of signs $\varepsilon_{i} \in\{ \pm 1\}$. Since $T_{i}^{-1}=T_{i}+\left(t_{i}^{-1}-t_{i}\right)$, this implies that

$$
Y^{\lambda}=\tau(\lambda) G^{ \pm}\left(\alpha^{(l)}\right) \ldots G^{ \pm}\left(\alpha^{(1)}\right)
$$

for some choice of the signs \pm , where $G^{+}(\alpha)=G(\alpha)$ and

$$
G^{-}(\alpha)=G(\alpha)-\left(t_{\alpha}-t_{\alpha}^{-1}\right) s_{\alpha}=\frac{t_{\alpha} X^{\alpha}-t_{\alpha}^{-1}}{X^{\alpha}-1}-\left(t_{\alpha}-t_{\alpha}^{-1}\right) \frac{X^{\alpha} s_{\alpha}}{X^{\alpha}-1} .
$$

These expressions for $T_{\tilde{w}}, Y^{\lambda}$ are rather complicated because the formula for $G^{ \pm}(\alpha)$ is a sum of two terms, one of which contains $s_{\alpha}$. We want to define some notion of "leading term" of $T_{\tilde{w}}$ in such a way that the terms with $s_{\alpha}$ from (5.1), (5.4) (or at least as many of them as possible) would not contribute to the leading term, thus making it easy to compute. 
We start with the definition of a new order on $P^{\vee}$.

Definition 5.1. Let $\lambda, \mu \in P^{\vee}$. We write $\lambda \lessdot \mu$ if

1. $\lambda^{+}<\mu^{+}$, where $\lambda^{+}$is the dominant coweight lying in the orbit of $\lambda$, and similarly for $\mu^{+}$,

or

2. $\lambda^{+}=\mu^{+}$and $\lambda>\mu$ (note that we are reversing the inequality!).

Note that this is only a partial order: there exist $\lambda, \mu$ that cannot be compared with respect to this order.

Proposition 5.2. Let $\tilde{w}=\tau(\lambda) w \in \widetilde{W}, w \in W$ and $\widehat{\alpha}=\alpha+k \delta \in R_{\tilde{w}}$ (see definition of $R_{\tilde{w}}$ in (5.2)). Write $\tilde{w} s_{\widehat{\alpha}}=\tau\left(\lambda^{\prime}\right) w^{\prime}, w^{\prime} \in W$. Then:

1. If $\widehat{\alpha} \in R$ (i.e., if $k=0$ ), then $\lambda^{\prime}=\lambda$.

2. If $\widehat{\alpha} \notin R$ (i.e., $k>0$ ), then $\lambda^{\prime} \lessdot \lambda$.

This proposition can be easily proved by direct calculation.

Let us define the notion of the leading term. Let $T$ be an operator on $\mathbb{C}_{q, t}[X]$ of the form

$$
T=\sum_{\lambda \in P^{\vee}, w \in W} g_{\lambda, w}(X) \tau(\lambda) w,
$$

where $g_{\lambda, w}$ are some rational functions in $X$.

Definition 5.3. Let $T$ be an operator of the form (5.5). Assume that it can be written in the following form:

$$
T=\sum_{w} g_{\lambda_{0}, w}(X) \tau\left(\lambda_{0}\right) w+\sum_{\lambda \lessdot \lambda_{0}, w} g_{\lambda, w}(X) \tau(\lambda) w
$$

for some $\lambda_{0}$ and that at least one of $g_{\lambda_{0}, w} \neq 0$. Then we say that $\sum_{w} g_{\lambda_{0}, w}(X) \tau\left(\lambda_{0}\right) w$ is the leading term of $T$ and denote it by $\langle T\rangle$.

Remark. Not every operator has a leading term. Also, it is not true that the leading term of a product is the product of the leading terms.

Example 5.4. Consider $Y^{\lambda}, \lambda \in P_{-}^{\vee}$. Then it is easy to see that $R_{\tau(\lambda)}$ contains only roots $\widehat{\alpha}=\alpha+k \delta$ with $k>0$. Also, by Theorem 3.7(2), all the signs in expression (5.3) should be -1 . Therefore, we have

$$
Y^{\lambda}=\tau(\lambda) G^{-}\left(\alpha^{(l)}\right) \ldots G^{-}\left(\alpha^{(1)}\right) .
$$

Substituting in this formula expression (5.4) for $G^{-}(\alpha)$ and using Proposition 5.2 and some simple arguments from the theory of affine Weyl groups, we see that the leading term of $Y^{\lambda}$ can be obtained by replacing each $G^{-}(\alpha)$ in the expression above by

$$
\frac{t_{\alpha} X^{\alpha}-t_{\alpha}^{-1}}{X^{\alpha}-1}
$$

in other words, by throwing away the term containing $s_{\alpha}$. Thus,

$$
\begin{aligned}
\left\langle Y^{\lambda}\right\rangle & =\tau(\lambda) \prod_{\alpha \in R_{\tau(\lambda)}} \frac{t_{\alpha} X^{\alpha}-t_{\alpha}^{-1}}{X^{\alpha}-1} \\
& =\left(\prod_{\alpha \in \tau(\lambda) R_{\tau(\lambda)}} \frac{t_{\alpha} X^{\alpha}-t_{\alpha}^{-1}}{X^{\alpha}-1}\right) \tau(\lambda)
\end{aligned}
$$


(note that this is a product of commuting expressions).

More generally, to find the leading term of $T_{\tilde{w}}$ we have to separate the affine and non-affine roots in $R_{\tilde{w}}$. Let us introduce the notations

$$
\begin{gathered}
R_{\tilde{w}}^{0}=R_{\tilde{w}} \cap R^{+}=\left\{\widehat{\alpha}=\alpha+k \delta \in R_{\tilde{w}} \mid k=0\right\} \\
R_{\tilde{w}}^{>0}=\left\{\widehat{\alpha}=\alpha+k \delta \in R_{\tilde{w}} \mid k>0\right\} .
\end{gathered}
$$

Lemma 5.5. For every $\tilde{w} \in \widetilde{W}$ there exists a reduced expression $\tilde{w}=\pi w, \pi \in$ $\widetilde{W}, w \in W$ such that $R_{\tilde{w}}^{0}=R_{w} .\left(\right.$ Thus, $R_{\tilde{w}}^{>0}=w^{-1} R_{\pi}$.)

Idea of proof. Take all reduced expressions $\tilde{w}=\pi w$ with $w \in W$ and choose the one with minimal $l(\pi)$; then use Lemma 3.2(2).

Corollary. It is possible to choose a reduced expression for $\tilde{w}$ in such a way that its associated sequence of roots $\alpha^{(i)}$ looks as follows:

$$
\underbrace{\alpha^{(l)}, \ldots, \alpha^{(k)}}_{R_{\widetilde{w}}^{>0}}, \underbrace{\alpha^{(k-1)}, \ldots, \alpha^{(1)}}_{R_{\tilde{w}}^{0}} .
$$

Theorem 5.6. 1. Let $\tilde{w}=\pi w$ be as in Lemma 5.5. Then

$$
\left\langle T_{\tilde{w}}\right\rangle=\left(\prod_{\alpha \in \tilde{w} R_{\tilde{w}}^{>0}} \frac{t_{\alpha} X^{\alpha}-t_{\alpha}^{-1}}{X^{\alpha}-1}\right) \pi T_{w} .
$$

2. Let $\lambda \in P^{\vee}$. Then

$$
\left\langle Y^{\lambda}\right\rangle=\sum_{w} g_{w}(X) \tau(\lambda) w
$$

for some rational functions $g_{w}$.

We could have made more precise statements about the leading term of $Y^{\lambda}$, but this is not necessary for our purposes.

Proof. (1) Write

$$
T_{\tilde{w}}=\tilde{w} G\left(\alpha^{(l)}\right) \ldots G\left(\alpha^{(k)}\right) G\left(\alpha^{(k-1)}\right) \ldots G\left(\alpha^{(1)}\right)
$$

where $\alpha^{(l)}, \ldots, \alpha^{(k)} \in R_{\tilde{w}}^{>0}, \alpha^{(k-1)}, \ldots, \alpha^{(1)} \in R_{\tilde{w}}^{0}$ (see the Corollary above). Then, due to Proposition 5.2, we have

$$
\begin{aligned}
\left\langle T_{\tilde{w}}\right\rangle & =\tilde{w}\left(\prod_{\alpha \in R_{\widetilde{w}}^{>0}} \frac{t_{\alpha} X^{\alpha}-t_{\alpha}^{-1}}{X^{\alpha}-1}\right) G\left(\alpha^{(k-1)}\right) \ldots G\left(\alpha^{(1)}\right) \\
& =\left(\prod_{\alpha \in \tilde{w} R_{\tilde{w}}^{>0}} \frac{t_{\alpha} X^{\alpha}-t_{\alpha}^{-1}}{X^{\alpha}-1}\right) \pi w G\left(\alpha^{(k-1)}\right) \ldots G\left(\alpha^{(1)}\right) \\
& =\left(\prod_{\alpha \in \tilde{w} R_{\tilde{w}}^{>0}} \frac{t_{\alpha} X^{\alpha}-t_{\alpha}^{-1}}{X^{\alpha}-1}\right) \pi T_{w} .
\end{aligned}
$$

(2) Follows from formula (5.3) for $Y^{\lambda}$ and Proposition 5.2. 
Theorem 5.7. The operators

$$
X^{\mu} T_{\tilde{w}}, \tilde{w} \in \widetilde{W}
$$

on $C_{q, t}[Y]$ are linearly independent.

Proof. It suffices to check that their leading terms are linearly independent. As for them, if $\tilde{w}=\tau(\lambda) w=\pi w^{\prime}, w, w^{\prime} \in W$ then

$$
\begin{aligned}
<X^{\mu} T_{\tau(\lambda) w}> & =X^{\mu} \prod_{\alpha \in \tilde{w} R_{\tilde{w}}^{>0}} \frac{t_{\alpha} X^{\alpha}-t_{\alpha}^{-1}}{X^{\alpha}-1} \pi T_{w^{\prime}} \\
& =X^{\mu} \prod_{\alpha \in \tilde{w} R_{\tilde{w}}^{>0}} \frac{t_{\alpha} X^{\alpha}-t_{\alpha}^{-1}}{X^{\alpha}-1} \tau(\lambda) w w^{\prime-1} T_{w^{\prime}} .
\end{aligned}
$$

Thus, it is easy to see that relation $\sum_{\lambda, \mu, w} a_{\lambda, \mu, w} X^{\mu} T_{\tau(\lambda) w}=0$ is possible only if $\sum_{\mu, w} a_{\lambda, \mu, w} X^{\mu} T_{\tau(\lambda) w}=0$. Since in the decomposition $\tilde{w}=\tau(\lambda) w=\pi w^{\prime}, \pi$ depends only on $\lambda$, the linear independence of $\left\langle X^{\mu} T_{\tau(\lambda) w}\right\rangle$ with fixed $\lambda$ follows from the fact that $T_{w}$ are linearly independent over the field of rational functions of $X$, which is based on the similar theorem for the affine Hecke algebra $\widehat{H}^{X}$ (Proposition 3.14).

Corollary 5.8. 1. Elements $X^{\mu} Y^{\lambda} T_{w}, \lambda \in P^{\vee}, \mu \in P, w \in W$, are linearly independent in $\mathcal{H}$.

2. $\mathbb{C}_{q, t}[X]$ is a faithful representation of $\mathcal{H}$.

3. The subalgebra $\widehat{H}^{Y} \subset \mathcal{H}$ spanned by $Y^{\lambda}, T_{w}$ is isomorphic to the affine Hecke algebra $\widehat{H}$ defined in Lecture 3 , and similarly for $\widehat{H}^{X}$.

Theorem 5.9. Let $\pi \in P^{\vee}$ be a minuscule coweight. Define $f_{\pi}=\sum_{w \in W} Y^{w(\pi)}$, and $L_{\pi}=\operatorname{Res} f_{\pi}$, where Res is defined by (4.5). Then

$$
L_{\pi}=\sum_{w \in W} w\left(\prod_{\substack{\alpha \in R \\(\alpha, \pi)=1}} \frac{t_{\alpha} X^{\alpha}-t_{\alpha}^{-1}}{X^{\alpha}-1} \tau(\pi)\right) .
$$

Proof. Let us calculate the leading term of $L_{\pi}$. It follows from the calculations of the leading term for $Y^{\lambda}$ (Example 5.4 and Theorem 5.6(2)) that $\left\langle L_{\pi}>\right.$ has the form $g(X) \tau\left(\pi^{-}\right)$, where $\pi^{-}$is the antidominant coweight lying in the orbit of $\pi: \pi^{-} \in P_{-}^{\vee}, \pi^{-} \in W \pi$. It follows from Theorem 5.6(2) that $\tau\left(\pi^{-}\right)$can come only from $Y^{\pi^{-}}$; using the calculation of the leading term for antidominant weight (Example 5.4), we see that the coefficient of $\tau\left(\pi^{-}\right)$is equal to

$$
\left|W_{\pi}\right| \prod_{\substack{\alpha \in R \\\left(\alpha, \pi^{-}\right)=1}} \frac{t_{\alpha} X^{\alpha}-t_{\alpha}^{-1}}{X^{\alpha}-1} \tau\left(\pi^{-}\right),
$$

where $W_{\pi}$ is the stabilizer of $\pi$ in $W$.

This gives us the leading term of $L_{\pi}$. Since $\pi$ is minuscule, it is known that there are no dominant coweights $\lambda$ with $\lambda<\pi$, and thus $\lambda \lessdot \pi^{-} \Longleftrightarrow \lambda \in W \pi$. Thus, $L_{\pi}$ contains only the terms of the form $g_{w}(X) \tau(w(\pi))$, which can be easily calculated, since we know one of them (with $\tau\left(\pi^{-}\right)$) and $L_{\pi}$ is $W$-invariant. This gives formula (5.9). 
Comparing formula (5.9) with the expression for Macdonald's difference operator $D_{\pi}$ defined in Lecture 2, we see that they coincide up to a constant factor. Thus, we see that the operators $L_{f}, f \in \mathbb{C}[Y]^{W}$ form a commutative algebra of difference operators which includes the Macdonald's difference operators - as was promised in Lecture 2.

\section{LECTURE 6: MACDONALD POLYNOMials REVISITED}

As usual, we start with recollections of some results of the previous lectures. Recall that we have defined the double affine Hecke algebra $\mathcal{H}$ which is generated by $X^{\mu}, \mu \in P, Y^{\lambda}, \lambda \in P^{\vee}, T_{w}, w \in W$. This algebra acts on the space $\mathbb{C}_{q, t}[X]$. Moreover, if $f \in \mathbb{C}_{q, t}[Y]^{W} \subset \mathcal{H}$, then the corresponding operator preserves the subspace $\mathbb{C}_{q, t}[X]^{W}$ and its restriction to this space coincides with some $W$-invariant difference operator $L_{f}$. Also, we checked that the $L_{f}$ 's commute and that if $\pi$ is a minuscule coweight, $f=\sum_{w} Y^{w(\pi)}$, then up to a constant factor, $L_{f}$ is the Macdonald's difference operator $D_{\pi}$ defined in Lecture 2 .

In this lecture we will prove that Macdonald polynomials are eigenfunctions of $L_{f}$ for any $f \in \mathbb{C}_{q, t}[Y]^{W}$. For simplicity, from now on we assume that $t_{\alpha}=q^{k_{\alpha}}$ for some $k_{\alpha} \in \mathbb{Z}_{+}$; thus, the field $\mathbb{C}_{q, t}$, considered in the previous lectures becomes $\mathbb{C}_{q}$. As before, the results of this lecture are due to Cherednik ([C6]).

Let us start with proving that $L_{f}$ are triangular in the basis of $m_{\lambda}$.

Definition. Define a partial order on $P$ as follows: $\lambda \prec \mu$ if $\lambda^{+}<\mu^{+}$or $\lambda^{+}=\mu^{+}$ and $\lambda<\mu$, where, as before, $\lambda^{+}$is the dominant weight lying in the orbit of $\lambda$.

Note that this order is similar to but not the same as the order $\lessdot$ on $P^{\vee}$ which we used in the previous lecture.

Lemma 6.1. Let $\lambda \in P^{\vee}$. Then the action of $\mathbb{C}_{t}[Y] \subset \mathcal{H}$ on $\mathbb{C}_{q}[X]$ has the following triangularity property:

$$
Y^{\lambda} X^{\mu}=\sum_{\nu \preceq \mu} c_{\mu \nu} X^{\nu}
$$

Also, if $\mu \in P^{+}$, then $c_{\mu \mu}=q^{2\left(\lambda, \mu+\rho_{k}\right)}$.

(Recall that $\rho_{k}=\frac{1}{2} \sum_{\alpha \in R^{+}} k_{\alpha} \alpha$.)

Proof. Assume first that $\lambda \in P_{+}^{\vee}$. Then the statement of the Lemma follows from the following two facts, which can be verified by direct calculation:

(1) $Y^{\lambda}=\tau(\lambda) G\left(\alpha^{(l)}\right) \ldots G\left(\alpha^{(1)}\right)$ (see $\left.(5.3)\right)$, where $\alpha^{(i)}$ run through the set $R_{\tau(\lambda)}=\left\{\widehat{\alpha}=\alpha+k \delta \mid \alpha \in R^{+}, 0 \leq k<(\lambda, \alpha)\right\}$

(2) If $\widehat{\alpha}=\alpha+k \delta$ is such that $\alpha \in R^{+}$, then

$$
G(\widehat{\alpha}) X^{\mu}=\left\{\begin{array}{lc}
t_{\alpha} X^{\mu}+\ldots & \text { if }\left(\mu, \alpha^{\vee}\right) \geq 0 \\
t_{\alpha}^{-1} X^{\mu}+\ldots & \text { if }\left(\mu, \alpha^{\vee}\right)<0
\end{array}\right.
$$

where the dots stand for a linear combination of $X^{\nu}$ with $\nu \prec \mu$.

If $\lambda$ is not dominant, we can write $Y^{\lambda}=Y^{\mu}\left(Y^{\nu}\right)^{-1}, \mu, \nu \in P_{+}^{\vee}$. Since the inverse of a triangular matrix is also triangular, the statement for $\lambda$ follows from the statements for $\mu, \nu$.

Lemma 6.1 immediately gives the triangularity of $L_{f}$. As before, let $m_{\mu}=$ $\sum_{\nu \in W \mu} X^{\nu}$ for $\mu \in P^{+}$be the basis of orbit-sums in $\mathbb{C}[X]^{W}$. We do not consider 
$m_{\mu}$ for non-dominant $\mu$, so whenever a formula contains $m_{\mu}$ it is always assumed that $\mu \in P^{+}$.

Theorem 6.2. Let $f \in \mathbb{C}_{q}[Y]^{W}, \mu \in P^{+}$. Then

$$
L_{f} m_{\mu}=f\left(q^{2\left(\mu+\rho_{k}\right)}\right) m_{\mu}+\sum_{\nu<\mu} a_{\mu \nu} m_{\nu}
$$

where, by definition, $f\left(q^{\mu}\right)$ is the polynomial in $q$ obtained by substituting $q^{(\lambda, \mu)}$ for $Y^{\lambda}$ in the expression for $f$.

Thus, it makes sense to talk about the eigenfunctions of $L_{f}$. Since a dominant weight $\mu$ is uniquely determined by the values $f\left(q^{\mu}\right)$ for all $f \in \mathbb{C}[Y]^{W}$, it is easy to see that for every dominant $\mu$ there exists a unique common eigenfunction of $L_{f}$ in $\mathbb{C}_{q}[X]^{W}$ with the highest term $X^{\mu}$. Later we will show that these eigenfunctions are nothing but Macdonald polynomials; this gives a new, uniform proof of the existence of Macdonald polynomials.

To prove that the eigenfunctions are Macdonald polynomials, we must check that they are orthogonal with respect to Macdonald's inner product. Recall that it was defined in Lecture 2 as follows: for $f, g \in \mathbb{C}_{q}[X]$ we let

$$
\langle f, g\rangle_{k}=\frac{1}{|W|}\left[f \bar{g} \Delta_{k}\right]_{0}
$$

where the $q$-linear bar involution is defined by $\overline{X^{\mu}}=X^{-\mu},[]_{0}$ is the constant term, and

$$
\Delta_{k}=\prod_{\alpha \in R} \prod_{i=0}^{k_{\alpha}-1}\left(1-q^{2 i} X^{\alpha}\right)
$$

This inner product is non-degenerate, $q$-linear, symmetric and $W$-invariant.

However, it turns out that we need to modify this inner product. Let us introduce the following involution on $\mathbb{C}_{q}: q^{\iota}=q^{-1}$ and extend it to $\mathbb{C}_{q}[X]$ by letting $\left(X^{\mu}\right)^{\iota}=$ $X^{\mu}$. Define the Cherednik's inner product by

$$
\langle f, g\rangle_{k}^{\prime}=\left[f \bar{g}^{\iota} \mu_{k}\right]_{0},
$$

where

$$
\mu_{k}=\prod_{\alpha \in R^{+}} \prod_{i=1-k_{\alpha}}^{k_{\alpha}}\left(q^{i} X^{\alpha / 2}-q^{-i} X^{-\alpha / 2}\right) .
$$

This inner product is neither symmetric nor $W$-invariant; still, it is better suited for our needs than Macdonald's inner product. Note that the weight function $\mu$ defined by (6.5) is very close to Macdonald's weight function $\Delta_{k}$. More precisely,

$$
\mu_{k}=(-1)^{\sum k_{\alpha}} q^{-\sum k_{\alpha}\left(k_{\alpha}-1\right)} \Delta_{k} \frac{\varphi_{k}}{\delta},
$$

where as before,

$$
\delta=\prod_{\alpha \in R^{+}}\left(X^{\alpha / 2}-X^{-\alpha / 2}\right),
$$

and

$$
\varphi_{k}=\prod_{\alpha \in R^{+}}\left(q^{k_{\alpha}} X^{\alpha / 2}-q^{-k_{\alpha}} X^{-\alpha / 2}\right) .
$$

Note also that $\bar{\mu}_{k}=\mu_{k}^{\iota}$. 
Proposition 6.3. Let $f, g \in \mathbb{C}_{q}[X]$. Then

1. $\langle f, g\rangle^{\prime}=\left(\langle g, f\rangle^{\prime}\right)^{\iota}$.

2. If $f, g \in \mathbb{C}_{q}[X]^{W}$, then

$$
\langle f, g\rangle_{k}^{\prime}=(-1)^{\sum k_{\alpha}} q^{-\sum k_{\alpha}\left(k_{\alpha}-1\right)} d_{k}\left\langle f, g^{\iota}\right\rangle_{k},
$$

where

$$
d_{k}=q^{\sum k_{\alpha}} \sum_{w \in W} q^{-2 \sum_{\alpha \in R_{w}} k_{\alpha}}
$$

Proof. (1) is trivial in view of $\bar{\mu}_{k}^{\iota}=\mu_{k}$. To prove (2), note that $\left[f \bar{g}^{\iota} \mu\right]_{0}=$ $\frac{1}{|W|} \sum_{w}\left[f \bar{g}^{\iota} w(\mu)\right]_{0}$, and the result follows from the identity

$$
\sum_{w \in W} w\left(\varphi_{k} / \delta\right)=\sum_{w} \prod_{\alpha \in R^{+}} q^{ \pm k_{\alpha}}=d_{k}
$$

where we take the sign "+" if $w(\alpha) \in R^{+}$and "_" otherwise. This identity can be proved in a standard way, by considering the highest term.

Remark. It is known (see [M4]) that $d_{k}$ can be written in the following form:

$$
d_{k}=\prod_{\alpha \in R^{+}} \frac{q^{\left(\alpha^{\vee}, \rho_{k}\right)+k_{\alpha}}-q^{-\left(\left(\alpha^{\vee}, \rho_{k}\right)+k_{\alpha}\right)}}{q^{\left(\alpha^{\vee}, \rho_{k}\right)}-q^{-\left(\alpha^{\vee}, \rho_{k}\right)}} .
$$

However, we will not use this formula.

Theorem 6.4. Let $P_{\lambda}, \lambda \in P_{+}$be the Macdonald polynomials (see Theorem 2.1). Then

1. $P_{\lambda}^{\iota}=P_{\lambda}$.

2. $\left\langle P_{\lambda}, P_{\mu}\right\rangle_{k}^{\prime}=0$ if $\lambda \neq \mu$.

3. $P_{\lambda}$ 's are uniquely defined by the triangularity condition $P_{\lambda}=m_{\lambda}+$ lower terms and the orthogonality condition (2) above.

Proof. (1) Since $\Delta^{\iota}=$ const $\cdot \Delta$, we see that $\left[P_{\lambda}^{\iota} \bar{P}_{\mu}^{\iota} \Delta\right]_{0}=0$ for $\mu \neq \lambda$, and thus $P_{\lambda}^{\iota}$ satisfy the definition of Macdonald polynomials.

(2) follows from (1) and the previous proposition; (3) is obvious since the Cherednik's inner product is non-degenerate.

Let us now define the notion of an adjoint operator. Let $h$ be an operator on $\mathbb{C}_{q}[X]$; define the corresponding adjoint operator $h^{*}$ by the condition

$$
\langle h f, g\rangle_{k}^{\prime}=\left\langle f, h^{*} g\right\rangle_{k}^{\prime} \text {. }
$$

An effective way to calculate adjoints is as follows. Define a simpler involution $h \mapsto h^{\dagger}$ by

$$
\left[h(f) \bar{g}^{\iota}\right]_{0}=\left[f{\overline{h^{\dagger}(g)}}^{\iota}\right]_{0} ;
$$

thus, $h^{\dagger}$ is the adjoint to $h$ with respect to the inner product $\langle,\rangle_{0}^{\prime}$. This adjoint is relatively easy to calculate; in particular,

$$
\begin{gathered}
p(X) \in \mathbb{C}_{q}[X] \Longrightarrow p^{\dagger}=\bar{p}^{\iota} \\
\tilde{w} \in \widetilde{W} \Longrightarrow \tilde{w}^{\dagger}=\tilde{w}^{-1}
\end{gathered}
$$

(this last condition justifies the introduction of $\iota$ in the definition of the inner product: otherwise it would not hold for $\tau(\lambda)$ ). 
On the other hand, the involutions $*$ and $\dagger$ are related by a simple rule

$$
h^{*}=\mu_{k}^{-1} h^{\dagger} \mu_{k},
$$

which obviously follows from the definitions. In particular, this implies that $p^{*}=\bar{p}^{\iota}$ for $p \in \mathbb{C}_{q}[X]$.

Theorem 6.5. 1. $T_{i}^{*}=T_{i}^{-1}$.

2. $\left(Y^{\lambda}\right)^{*}=Y^{-\lambda}$.

Proof. (1) Since $T_{i}^{-1}=T_{i}+\left(t_{i}^{-1}-t_{i}\right), t_{i}^{*}=t_{i}^{-1}$, it suffices to prove that $\left(T_{i}-t_{i}\right)^{*}=$ $T_{i}-t_{i}$. From the definition of the action of $T_{i}$ we get by direct calculation that

$$
T_{i}-t_{i}=\frac{t_{i} X^{-\alpha_{i} / 2}-t_{i}^{-1} X^{\alpha_{i} / 2}}{X^{-\alpha_{i} / 2}-X^{\alpha_{i} / 2}}\left(s_{i}-1\right) .
$$

Since

$$
s_{i}^{*}=\mu^{-1} s_{i} \mu=-\varphi_{k}^{-1} s_{i} \varphi_{k}=\frac{t_{i}^{-1} X^{\alpha_{i} / 2}-t_{i} X^{-\alpha_{i} / 2}}{t_{i} X^{\alpha_{i} / 2}-t_{i}^{-1} X^{-\alpha_{i} / 2}} s_{i}
$$

we get

$$
\begin{aligned}
\left(T_{i}-t_{i}\right)^{*} & =\left(s_{i}^{*}-1\right) \frac{t_{i}^{-1} X^{\alpha_{i} / 2}-t_{i} X^{-\alpha_{i} / 2}}{X^{\alpha_{i} / 2}-X^{-\alpha_{i} / 2}} \\
& =\frac{t_{i}^{-1} X^{\alpha_{i} / 2}-t_{i} X^{-\alpha_{i} / 2}}{X^{\alpha_{i} / 2}-X^{-\alpha_{i} / 2}}\left(s_{i}-1\right)=T_{i}-t_{i} .
\end{aligned}
$$

(2) It suffices to prove this formula for $\lambda \in P_{+}^{\vee}$, in which case it follows from Theorem 3.7, the previous statement and $\pi^{*}=\pi^{-1}, \pi \in \Omega$, which again can be proved by direct calculation.

This means that we can consider ${ }^{*}$ as an involution on $\mathbb{C}_{q}[Y]$ which is defined by $\left(f(q) Y^{\lambda}\right)^{*}=f^{\iota} Y^{-\lambda}$; in particular, this involution preserves $\mathbb{C}_{q}[Y]^{W}$.

Theorem 6.6. Macdonald polynomials are eigenfunctions of $L_{f}, f \in \mathbb{C}_{q}[Y]^{W}$ :

$$
L_{f} P_{\lambda}=f\left(q^{2\left(\lambda+\rho_{k}\right)}\right) P_{\lambda} .
$$

Proof. Since $\left.L_{f}\right|_{\mathbb{C}_{q}[X]^{W}}=\left.f\right|_{\mathbb{C}_{q}[X]^{W}}$, it suffices to prove that Macdonald polynomials are eigenfunctions of $f \in \mathbb{C}_{q}[Y]^{W}$. Since ${ }^{*}$ preserves $\mathbb{C}_{q}[Y]^{W}$, Theorem 6.2 implies that both $f$ and $f^{*}$ are upper-triangular in the basis of $P_{\lambda}$. On the other hand, by Theorem 6.4, $P_{\lambda}$ 's are orthogonal with respect to the Cherednik's inner product. This is only possible if $f$ is diagonal. The eigenvalues are given by (6.1).

Proposition 6.7. The operators $L_{f}, f \in \mathbb{C}_{q}[Y]^{W}$ are self-adjoint with respect to Macdonald's inner product.

Proof. It suffices to prove that their restrictions to $\mathbb{C}_{q}[X]^{W}$ are self-adjoint, which follows from the fact that they are diagonalized in the basis of Macdonald polynomials.

This completes a large part of this course: we have constructed a commuting family of difference operators, whose eigenfunctions are the Macdonald polynomials. In the next lecture we will use this construction to prove the inner product identities. 


\section{Lecture 7: Proof of Macdonald's inner Product identities}

Recall that we have defined an action of the double affine Hecke algebra $\mathcal{H}$ on the space $\mathbb{C}_{q}[X]$. Also, we have defined Cherednik's inner product $\langle,\rangle_{k}^{\prime}$ on $\mathbb{C}_{q}[X]$ such that with respect to this inner product $\left(Y^{\lambda}\right)^{*}=Y^{-\lambda}$, and on symmetric functions $\langle,\rangle_{k}^{\prime}$ coincides up to a factor with Macdonald's inner product $\langle,\rangle_{k}$.

In this lecture we will use the action of $\mathcal{H}$ to prove Macdonald's inner product identities (see Theorem 2.4). From now on, we assume for simplicity that all $k_{\alpha}$ 's are equal: $k_{\alpha}=k$, so that all $t_{\alpha}=t=q^{k}$. In fact, it is not much more difficult to repeat all the arguments in the general case; later we will outline the necessary changes.

The proof presented here is due to Cherednik. However, our exposition follows that of Macdonald ([M5]), who simplified the original arguments of Cherednik. For example, the operator $\widehat{G}$ below was introduced by Macdonald.

The idea of the proof is quite simple. First note that due to Proposition 6.3, the calculation of $\left\langle P_{\lambda}, P_{\lambda}\right\rangle_{k}$ is equivalent to the calculation of $\left\langle P_{\lambda}, P_{\lambda}\right\rangle_{k}^{\prime}$. Using the large set of operators we have constructed, we want to prove the theorem by induction on $k$. Let us write $P_{\lambda}^{(k)}$ to stress the dependence of Macdonald polynomials on $k$. We want to construct some operator $G: \mathbb{C}_{q}[X]^{W} \rightarrow \mathbb{C}_{q}[X]^{W}$ (shift operator), which would shift $k \rightarrow k+1$. More precisely, we want:

(1) $G P_{\lambda+\rho}^{(k)}=$ const $\cdot P_{\lambda}^{(k+1)}$ for some easily computable constant.

(2) $\langle G f, g\rangle_{k+1}^{\prime}=\langle f, \widehat{G} g\rangle_{k}^{\prime}$ for some operator $\widehat{G}$.

If we construct such an operator, then the calculation of $\left\langle P_{\lambda}^{(k+1)}, P_{\lambda}^{(k+1)}\right\rangle_{k+1}^{\prime}$ can be reduced to the calculation of $\left\langle P_{\lambda+\rho}^{(k)}, \widehat{G} G P_{\lambda+\rho}^{(k)}\right\rangle_{k}^{\prime}$, or - if we know the diagonal entries of $\widehat{G} G$ - to the calculation of $\left\langle P_{\lambda+\rho}^{(k)}, P_{\lambda+\rho}^{(k)}\right\rangle_{k}^{\prime}$. Repeating the process, we reduce the question to the calculation of $\left\langle P_{\lambda+k \rho}^{(0)}, P_{\lambda+k \rho}^{(0)}\right\rangle_{0}^{\prime}$, which is trivial.

In the differential $(q=1)$ case, the shift operators were introduced by Opdam (see [O3], [H3]). The construction for arbitrary $q$ described below is due to Cherednik $([\mathrm{C} 6])$.

To define $G, \widehat{G}$, we need the following operators:

$$
\begin{gathered}
\mathcal{X}=\varphi_{-k}=\prod_{\alpha \in R^{+}}\left(q^{-k} X^{\alpha / 2}-q^{k} X^{-\alpha / 2}\right) \\
\mathcal{Y}=\varphi_{-k}^{\vee}(Y)=\prod_{\alpha \in R^{+}}\left(q^{-k} Y^{\alpha^{\vee} / 2}-q^{k} Y^{-\alpha^{\vee} / 2}\right) \\
\widehat{\mathcal{Y}}=\varphi_{k}^{\vee}(Y)=\prod_{\alpha \in R^{+}}\left(q^{k} Y^{\alpha^{\vee} / 2}-q^{-k} Y^{-\alpha^{\vee} / 2}\right) .
\end{gathered}
$$

It is easily seen from the previous results that $\mathcal{X}^{\iota}=(-1)^{\left|R^{+}\right|} \overline{\mathcal{X}}=\varphi_{k}, \mathcal{X}^{*}=$

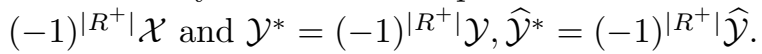

Now, define the shift operators by

$$
G=\mathcal{X}^{-1} \mathcal{Y}, \quad \widehat{G}=\widehat{\mathcal{Y}} \mathcal{X} .
$$

Theorem 7.1. G, $\widehat{G}$ preserve $\mathbb{C}_{q}[X]^{W}$.

Proof. Recall that $f \in \mathbb{C}_{q}[X]^{W} \Longleftrightarrow\left(T_{i}-t\right) f=0$ for all $i$ (this follows, for example, from formula $(6.10))$. Define $\mathbb{C}_{q}[X]^{-W}=\left\{f \in \mathbb{C}_{q}[X] \mid\left(T_{i}+t^{-1}\right) f=0\right\}$. It is easy to see that as $q \rightarrow 1$, this definition becomes the usual definition of 
anti-invariant polynomials. Now, to prove the theorem it suffices to prove that $\mathcal{X}\left(\mathbb{C}_{q}[X]^{W}\right)=\mathbb{C}_{q}[X]^{-W}, \mathcal{Y}\left(\mathbb{C}_{q}[X]^{W}\right) \subset \mathbb{C}_{q}[X]^{-W}, \widehat{\mathcal{Y}}\left(\mathbb{C}_{q}[X]^{-W}\right) \subset \mathbb{C}_{q}[X]^{W}$. In fact, once we prove that $\mathcal{X}\left(\mathbb{C}_{q}[X]^{W}\right) \subset \mathbb{C}_{q}[X]^{-W}$, the statement that this embedding is an isomorphism can be easily proved by deformation arguments, since in the limit $q \rightarrow 1$ this statement is well known. Thus, the theorem follows from the lemma below.

\section{Lemma.}

$$
\begin{aligned}
& \left(T_{i}+t^{-1}\right) \mathcal{X}=\frac{t^{-1} X^{-\alpha_{i} / 2}-t X^{\alpha_{i} / 2}}{t^{-1} X^{\alpha_{i} / 2}-t X^{-\alpha_{i} / 2}} \mathcal{X}\left(T_{i}-t\right) \\
& \left(T_{i}+t^{-1}\right) \mathcal{Y}=\frac{t^{-1} Y^{-\alpha_{i}^{\vee} / 2}-t Y^{\alpha_{i}^{\vee} / 2}}{t^{-1} Y^{\alpha_{i}^{\vee} / 2}-t Y^{-\alpha_{i}^{\vee} / 2}} \mathcal{Y}\left(T_{i}-t\right) \\
& \left(T_{i}-t\right) \hat{\mathcal{Y}}=\frac{t Y^{-\alpha_{i}^{\vee} / 2}-t^{-1} Y^{\alpha_{i}^{\vee} / 2}}{t Y^{\alpha_{i}^{\vee} / 2}-t^{-1} Y^{-\alpha_{i}^{\vee} / 2}} \widehat{\mathcal{Y}}\left(T_{i}+t^{-1}\right) .
\end{aligned}
$$

This lemma is proved by direct calculation, using the identity $T_{i} X^{\alpha_{i} / 2}-X^{-\alpha_{i} / 2} T_{i}$ $=\left(t-t^{-1}\right) X^{\alpha_{i} / 2}$.

Now we can formulate the main property of the shift operators.

Theorem 7.2. Let $f, g \in \mathbb{C}_{q}[X]^{W}$. Then

$$
\langle G f, g\rangle_{k+1}^{\prime}=\frac{d_{k+1}}{d_{k}}\langle f, \widehat{G} g\rangle_{k}^{\prime}
$$

where $d_{k}$ is defined in Proposition 6.3.

Remark. Since $\mu_{k+1}=\varphi_{k+1} \varphi_{-k} \mu_{k}$, it is easy to see that $\langle G f, g\rangle_{k+1}^{\prime}=\left\langle f, \mathcal{Y} \varphi_{k+1} g\right\rangle_{k}^{\prime}$. But this is of little use, since $\mathcal{Y} \varphi_{k+1}$ does not preserve $\mathbb{C}_{q}[X]^{W}$; thus, to find, say, $\left\langle P_{\lambda}, \mathcal{Y} \varphi_{k+1} m_{\mu}\right\rangle_{k}^{\prime}$ we have to calculate the highest term of the projection of $\mathcal{Y} \varphi_{k+1} m_{\mu}$ on $\mathbb{C}_{q}[X]^{W}$, which is very difficult.

Proof. The proof is based on the following simple idea, which we have already used before. Let $\mathcal{P}=\frac{1}{|W|} \sum_{w \in W} w$ be the usual symmetrizer. Then for every $f \in \mathbb{C}_{q}[X]$ we have $[f]_{0}=[\mathcal{P} f]_{0}$. Thus, if $\mathcal{P} f=\mathcal{P} g$, then $[f]_{0}=[g]_{0}$. Also, we will need the following proposition.

Proposition 7.3. Let $\mathcal{P}_{-}=\frac{1}{|W|} \sum_{w \in W}(-1)^{l(w)} w$ be the usual antisymmetrizer. Then for every $f \in \mathbb{C}_{q}[X]^{W}$ we have

$$
\mathcal{P}_{-} \mathcal{Y} f=\mathcal{P}_{-} \widehat{\mathcal{Y}} f
$$

The proof of this proposition is non-trivial and requires an introduction of a new interesting operator - the $q$-antisymmetrizer. We will give this proof in the next lecture.

Now let us prove the theorem. By definition,

$$
\langle G f, g\rangle_{k+1}^{\prime}=\left[(G f) \bar{g}^{\iota} \mu_{k+1}\right]_{0}=\left[(G f) \bar{g}^{\iota} \mathcal{P}\left(\mu_{k+1}\right)\right] .
$$

Since $\mu_{k+1}=\varphi_{k+1} \varphi_{-k} \mu_{k}=\varphi_{k+1} \mathcal{X} \mu_{k}$, and $\mathcal{X} \mu_{k}$ is antisymmetric (this is the crucial step!), we have $\mathcal{P}\left(\mu_{k+1}\right)=\mathcal{P}_{-}\left(\varphi_{k+1}\right) \mathcal{X} \mu_{k}=\frac{1}{|W|} d_{k+1} \delta \mathcal{X} \mu_{k}$ (see formula $(6.8))$. 
Similarly, $\mathcal{P}\left(\mathcal{X}^{2} \mu_{k}\right)=\frac{1}{|W|} d_{k} \delta \mathcal{X} \mu_{k}$, and thus,

$$
\mathcal{P}\left(\mu_{k+1}\right)=\frac{d_{k+1}}{d_{k}} \mathcal{P}\left(\mathcal{X}^{2} \mu_{k}\right) .
$$

Substituting this in the expression for $\langle G f, g\rangle_{k+1}^{\prime}$, we get

$$
\begin{aligned}
\langle G f, g\rangle_{k+1}^{\prime} & =\frac{d_{k+1}}{d_{k}}\left[\mathcal{X}^{-1} \mathcal{Y}(f) \bar{g}^{\iota} \mathcal{X}^{2} \mu_{k}\right]_{0} \\
& =\frac{d_{k+1}}{d_{k}}\left[\mathcal{P}\left(\mathcal{Y}(f) \bar{g}^{\iota} \mathcal{X} \mu_{k}\right)\right]_{0}=\frac{d_{k+1}}{d_{k}}\left[\mathcal{P}_{-}(\mathcal{Y}(f)) \bar{g}^{\iota} \mathcal{X} \mu_{k}\right]_{0}
\end{aligned}
$$

Using Proposition 7.3, we can replace in the last formula $\mathcal{Y}$ by $\widehat{\mathcal{Y}}$, and thus

$$
\begin{aligned}
\langle G f, g\rangle_{k+1}^{\prime} & =\frac{d_{k+1}}{d_{k}}\left[\mathcal{X} \widehat{\mathcal{Y}}(f) \bar{g}^{\iota} \mu_{k}\right]_{0} \\
& =\frac{d_{k+1}}{d_{k}}\langle\mathcal{X} \widehat{\mathcal{Y}} f, g\rangle_{k}^{\prime}=\frac{d_{k+1}}{d_{k}}\langle f, \widehat{\mathcal{Y}} \mathcal{X}\rangle_{k}^{\prime},
\end{aligned}
$$

which completes the proof of Theorem 7.2.

This theorem immediately implies that the shift operators indeed shift the parameter $k$ of Macdonald polynomials:

Theorem 7.4. $\quad$ 1. $G P_{\lambda+\rho}^{(k)}=\left.q^{k \mid R^{+}}\right|_{c_{k}}(\lambda) P_{\lambda}^{(k+1)}$, where

$$
c_{k}(\lambda)=\prod_{\alpha \in R^{+}}\left(q^{-k+\left(\alpha^{\vee}, \lambda+(k+1) \rho\right)}-q^{k-\left(\alpha^{\vee}, \lambda+(k+1) \rho\right)}\right),
$$

$$
\text { and } G P_{\lambda}^{(k)}=0 \text { if } \lambda-\rho \notin P_{+} \text {. }
$$

2. For $k \geq 0, \widehat{G} P_{\lambda}^{(k+1)}=q^{-k\left|R^{+}\right|} \hat{c}_{k}(\lambda) P_{\lambda+\rho}^{(k)}$, where

$$
\hat{c}_{k}(\lambda)=\prod_{\alpha \in R^{+}}\left(q^{k+\left(\alpha^{\vee}, \lambda+(k+1) \rho\right)}-q^{-k-\left(\alpha^{\vee}, \lambda+(k+1) \rho\right)}\right) .
$$

Proof. First, it is easy to prove, using Lemma 6.1, that $G P_{\lambda+\rho}^{(k)}=q^{k\left|R^{+}\right|} c_{k} m_{\lambda}+\ldots$. Thus, to prove (1) it suffices to check that $\left\langle G P_{\lambda+\rho}^{(k)}, m_{\mu}\right\rangle_{k+1}^{\prime}=0$ if $\mu<\lambda$. Due to Theorem 7.2 , this is equivalent to $\left\langle P_{\lambda+\rho}^{(k)}, \widehat{G} m_{\mu}\right\rangle_{k}^{\prime}=0$. Since $\widehat{G} m_{\mu}$ is a linear combination of $m_{\nu}$ with $\nu \leq \mu+\rho$ (this also follows from Lemma 6.1), the statement follows from the definition of Macdonald polynomials. (2) is proved in a similar way.

Now we can prove Macdonald's inner product identities. Let us introduce $M_{k}^{\prime}(\lambda)=\left\langle P_{\lambda}^{(k)}, P_{\lambda}^{(k)}\right\rangle_{k}^{\prime}$.

\section{Proposition 7.5.}

$$
M_{k+1}^{\prime}(\lambda)=(-1)^{\left|R^{+}\right|} \frac{d_{k+1}}{d_{k}} \frac{\hat{c}_{k}(\lambda)}{c_{k}(\lambda)} M_{k}^{\prime}(\lambda+\rho) .
$$


Proof. Using the previous theorem, we can write

$$
\begin{aligned}
M_{k+1}^{\prime}(\lambda) & =\left(c_{k}(\lambda) c_{k}(\lambda)^{\iota}\right)^{-1}\left\langle G P_{\lambda+\rho}^{(k)}, G P_{\lambda+\rho}^{(k)}\right\rangle_{k+1}^{\prime} \\
& =\frac{d_{k+1}}{d_{k}}\left(c_{k}(\lambda) c_{k}(\lambda)^{\iota}\right)^{-1}\left\langle P_{\lambda+\rho}^{(k)}, \widehat{G} G P_{\lambda+\rho}^{(k)}\right\rangle_{k}^{\prime} \\
& =\frac{\left(\hat{c}_{k}(\lambda) c_{k}(\lambda)\right)^{\iota}}{c_{k}(\lambda) c_{k}(\lambda)^{\iota}} \frac{d_{k+1}}{d_{k}}\left\langle P_{\lambda+\rho}^{(k)}, P_{\lambda+\rho}^{(k)}\right\rangle_{k}^{\prime}=(-1)^{\left|R^{+}\right|} \frac{d_{k+1}}{d_{k}} \frac{\hat{c}_{k}(\lambda)}{c_{k}(\lambda)} M_{k}^{\prime}(\lambda+\rho) .
\end{aligned}
$$

Corollary 7.6. Let $M_{k}(\lambda)=\left\langle P_{\lambda}^{(k)}, P_{\lambda}^{(k)}\right\rangle_{k}=d_{k}^{-1}(-1)^{k\left|R^{+}\right|} q^{k(k-1)\left|R^{+}\right|} M_{k}^{\prime}(\lambda)$ (see Proposition 6.3). Then

$$
M_{k+1}(\lambda)=\prod_{\alpha \in R^{+}} \frac{1-q^{2\left(\alpha^{\vee}, \lambda+(k+1) \rho\right)+2 k}}{1-q^{2\left(\alpha^{\vee}, \lambda+(k+1) \rho\right)-2 k}} M_{k}(\lambda+\rho) .
$$

Applying this corollary $k-1$ times and using $M_{1}(\lambda)=1$ for all $\lambda \in P_{+}$(this is equivalent to saying that the Weyl characters are orthonormal), we get the Macdonald's inner product identities, formulated in Lecture 2:

Theorem 7.7. (Macdonald's inner product identities) If all $k_{\alpha}=k$, then

$$
\left\langle P_{\lambda}, P_{\lambda}\right\rangle_{k}=\prod_{\alpha \in R^{+}} \prod_{i=1}^{k-1} \frac{1-q^{2\left(\alpha^{\vee}, \lambda+k \rho\right)+2 i}}{1-q^{2\left(\alpha^{\vee}, \lambda+k \rho\right)-2 i}} .
$$

To prove the inner product identities in the case where $k_{\alpha}$ are not necessarily equal (see Theorem 2.4), we have to introduce the shift operators separately for the long and short roots. They are defined in precisely the same way as before, but with the product in (7.1) taken only over long (respectively, short) roots. Repeating the steps above with necessary changes, we can prove that the shift operators for long roots (respectively, short roots) shift the corresponding $k_{\alpha}$ by one. This gives the proof of the general Macdonald's inner product identities (2.6). We refer the reader to [C6] for details.

\section{LECTURE 8: $q$-SYMMETRIZERS}

In this lecture we prove Proposition 7.3 and thus complete the proof of the inner product identities. Recall that we want to prove $\mathcal{P}_{-} \mathcal{Y} f=\mathcal{P}_{-} \widehat{\mathcal{Y}} f$ for every $f \in \mathbb{C}_{q}[X]^{W}$, where $\mathcal{P}_{-}$is the antisymmetrizer and $\mathcal{Y}, \widehat{\mathcal{Y}}$ are defined by (7.1). Unfortunately, the commutation relations between $w \in W$ and $Y^{\lambda}$ are very complicated, making direct calculation impossible. However, there is a bypass, which involves the introduction of the so-called " $q$-antisymmetrizer". This does not seem to be closely related with Macdonald's theory, but it is interesting enough in itself, so we spend some time discussing these new operators.

Let us start with describing the kernel of the antisymmetrizer.

Theorem 8.1. In any finite-dimensional representation $V$ of $W$ we have

$$
\operatorname{Ker} \mathcal{P}_{-}=\sum_{i} \operatorname{Ker}\left(1-s_{i}\right)
$$

Proof. Denote $V_{i}=\operatorname{Ker}\left(1-s_{i}\right), V^{\prime}=\sum V_{i}$.

Lemma. $V^{\prime}$ is $W$-invariant. 
Proof. It suffices to prove $s_{i} V_{j} \subset V_{i}+V_{j}$. Let $v \in s_{i} V_{j}$; then $s_{j}\left(s_{i} v\right)=s_{i} v$. Introduce $v_{ \pm}=\frac{1}{2}\left(v \pm s_{i} v\right)$. Then $v=v_{+}+v_{-}, s_{i} v=v_{+}-v_{-}$, and thus $s_{j}\left(v_{+}-v_{-}\right)=$ $v_{+}-v_{-}$, so $v_{+}-v_{-} \in V_{j}$. Since by definition $v_{+} \in V_{i}$, we see that $v \in V_{i}+V_{j}$.

Let us return to the proof of the theorem. Obviously, it suffices to prove this theorem for an irreducible representation. In this case, $V^{\prime}$ can be either 0 or $V$. But:

$$
\begin{gathered}
V^{\prime}=0 \Longleftrightarrow \text { all } V_{i}=0 \Longleftrightarrow \\
\text { for all } i, \quad\left(1-s_{i}\right) \text { is invertible } \Longleftrightarrow \\
\text { for all } i, \quad s_{i}=-1 \text { in } V \Longleftrightarrow \\
V \text { is the sign representation. }
\end{gathered}
$$

Thus, for an irreducible $V$ we have

$$
V^{\prime}= \begin{cases}0, & \text { if } V \text { is the sign representation } \\ V & \text { otherwise. }\end{cases}
$$

This coincides with Ker $\mathcal{P}_{-}$.

Note that (8.1) also holds for the representation of $W$ in the space of polynomials $\mathbb{C}_{q}[X]$, since this representation is a direct sum of finite-dimensional representations.

The main idea of the proof of Proposition 7.3 is to describe $\operatorname{Ker} \mathcal{P}_{-}$in $\mathbb{C}_{q}[X]$ using the action of the Hecke algebra $H$ generated by $T_{1}, \ldots, T_{n}$ rather than the action of $W$, and then use the commutation relations of $H$ with $Y$.

Let us introduce the following element of $H$, which we will call the $q$-antisymmetrizer:

$$
\mathcal{P}_{-}^{q}=d^{-1} \sum_{w \in W}(-t)^{-l(w)} T_{w}
$$

where $d=\sum_{w \in W} t^{-2 l(w)}$.

It is easy to see that as $q \rightarrow 1$ this element becomes the usual antisymmetrizer $\mathcal{P}_{-}$.

Theorem 8.2. 1. For every $i=1, \ldots, n, \mathcal{P}_{-}^{q}$ is divisible by $T_{i}-t$ both on the left and on the right.

2. We have the following properties for the action of $\mathcal{P}_{-}^{q}$ on $\mathbb{C}_{q}[X]$ :

$$
\begin{gathered}
\operatorname{Ker} \mathcal{P}_{-}^{q}=\operatorname{Ker} \mathcal{P}_{-} \\
\operatorname{Im} \mathcal{P}_{-}^{q}=\mathbb{C}_{q}[X]^{-W}=\left\{f \in \mathbb{C}_{q}[X] \mid\left(T_{i}+t^{-1}\right) f=0\right\} .
\end{gathered}
$$

3. $\mathcal{P}_{-}^{q}$ is a projector.

Proof. (1) Since $w \mapsto w s_{i}$ is an involution of the Weyl group, $W$ is a union of pairs $w, w s_{i}$ where $w$ is such that $l\left(w s_{i}\right)=l(w)+1$. Thus,

$$
\mathcal{P}_{-}^{q}=d^{-1} \sum_{l\left(w s_{i}\right)=l(w)+1}(-t)^{-l(w)} T_{w}\left(1-t^{-1} T_{i}\right) .
$$

Divisibility on the left is proved similarly.

(2) It follows from (1) that $\operatorname{Ker} \mathcal{P}_{-}^{q} \supset \sum \operatorname{Ker}\left(T_{i}-t\right)=\sum \operatorname{Ker}\left(s_{i}-1\right)$. On the other hand, for $q=1$ this inclusion is an equality by Theorem 8.1. Since the rank of an operator cannot increase under specialization, we see that $\operatorname{Ker} \mathcal{P}_{-}^{q}=\operatorname{Ker} \mathcal{P}_{-}=$ $\sum \operatorname{Ker}\left(1-s_{i}\right)$. Similarly, (1) implies that $\operatorname{Im} \mathcal{P}_{-}^{q} \subset \mathbb{C}_{q}[X]^{-W}$; since the dimension of $\mathbb{C}_{q}[X]^{-W}$ is independent of $q$, we see that $\operatorname{Im} \mathcal{P}_{-}^{q}=\mathbb{C}_{q}[X]^{-W}$. (Of course, to 
make sense of these dimension arguments we must consider $\mathbb{C}_{q}[X]$ as a filtered space and note that both $\mathcal{P}_{-}$and $\mathcal{P}_{-}^{q}$ preserve this filtration.)

(3) is trivial: let $v \in \mathbb{C}_{q}[X]^{-W}$. Then $T_{i} v=-t^{-1} v$, so $T_{w} v=(-t)^{-l(w)} v$, and $\mathcal{P}_{-}^{q} v=v$.

Corollary. For $f \in \mathbb{C}_{q}[X], \mathcal{P}_{-} f=0 \Longleftrightarrow \mathcal{P}_{-}^{q} f=0$.

Thus, to prove Proposition 7.3 it suffices to prove $\mathcal{P}_{-}^{q}(\mathcal{Y}-\widehat{\mathcal{Y}}) f=0$ for every $f \in \mathbb{C}_{q}[X]^{W}$. Using the fact that $\mathbb{C}_{q}[X]$ is a faithful representation of $\mathcal{H}$, it is easy to prove that this last condition is equivalent to

$$
\mathcal{P}_{-}^{q}(\mathcal{Y}-\widehat{\mathcal{Y}})=\sum h_{i}\left(T_{i}-t\right) \quad \text { for some } h_{i} \in \widehat{H}^{Y}
$$

as elements of $\widehat{H}^{Y}$.

Now, we can do the following trick. Since (8.3) is an identity in $\widehat{H}^{Y}$, it suffices to prove it in any faithful representation of $\widehat{H}^{Y}$. Let us prove it in $\mathbb{C}_{q}[Y]$ (see Lecture 3). Now we can repeat the same chain of arguments in reverse order: (8.3) $\Longleftrightarrow \mathcal{P}_{-}^{q}(\mathcal{Y}-\widehat{\mathcal{Y}}) f=0$ for every $f \in \mathbb{C}_{q}[Y]^{W} \Longleftrightarrow \mathcal{P}_{-}(\mathcal{Y}-\widehat{\mathcal{Y}}) f=0$. But this last condition is trivial: since $\mathcal{Y}, \widehat{\mathcal{Y}}$ act on $\mathbb{C}_{q}[Y]$ by multiplication, and $W$ acts by $w: Y^{\lambda} \mapsto Y^{w(\lambda)}$, we have $w_{0}(\mathcal{Y})=(-1)^{\left|R^{+}\right|} \widehat{\mathcal{Y}}, w_{0}(\widehat{\mathcal{Y}})=(-1)^{\left|R^{+}\right|} \mathcal{Y}$, where $w_{0}$ is the longest element of the Weyl group. Since $\mathcal{P}_{-}$is divisible by $\left(1+(-1)^{\left|R^{+}\right|} w_{0}\right)$, we have $\mathcal{P}_{-}(\mathcal{Y}-\widehat{\mathcal{Y}})=0$. This completes the proof of Proposition 7.3, and thus, the proof of Macdonald's inner product identities.

\section{REFERENCES}

[AI] Askey, R. and Ismail, M.E.H., A generalization of ultraspherical polynomials, Studies in Pure Mathematics (P. Erdös, ed.), Birkhäuser, 1982, pp. 55-78. MR 87a:33015

[AW] Askey, R. and Wilson, J., Some basic hypergeometric orthogonal polynomials that generalize Jacobi polynomials, Memoirs of AMS 319 (1985). MR 87a:05023

[B] Bourbaki, N., Groupes et algèbres de Lie, Ch. 4-6, Hermann, Paris, 1969. MR 39:1590

[BZ] Bressoud, D. and Zeilberger, D., A proof of Andrews' q-Dyson conjecture, Discrete Math. 54 (1985), 201-224. MR 87f:05015

[C1] Cherednik, I., Double affine Hecke algebras, Knizhnik-Zamolodchikov equations, and Macdonald's operators, IMRN (Duke M.J.) 9 (1992), 171-180. MR 94b:17040

[C2] , The Macdonald constant term conjecture, IMRN 6 (1993), 165-177. MR 94i:17016

[C3] _ A unification of Knizhnik-Zamolodchikov and Dunkl operators via affine Hecke algebras, Inventiones Math. 106 (2) (1991), 411-432. MR 93b:17040

[C4] - Quantum Knizhnik-Zamolodchikov equations and affine root systems, Commun. Math. Phys. 150 (1992), 109-136. MR 94a:17019

[C5] - Integration of Quantum many-body problems by affine Knizhnik-Zamolodchikov equations, Advances in Math. 106 (1994), 65-95. MR 95m:32031

[C6] , Double affine Hecke algebras and Macdonald's conjectures, Annals of Math. 141 (1995), 191-216. MR 96m:33010

[C7] , Macdonald's evaluation conjectures and difference Fourier transform, Invent. Math. 122 (1995), 119-145. MR 1:354 956

[CRM] Calogero, P., Ragnisco, O., and Marchioro, C., Exact solution of the classical and quantal one-dimensional many-body problems with the two-body potential $V_{a}(x)=$ $g^{2} a^{2} / \sinh ^{2}(a x)$, Lett. Nuovo Cimento 13 (1975), 383-387. MR 56:2029

[D] van Diejen, J.F., Commuting difference operators with polynomial eigenfunctions, Compos. Math. 95 (1995), 183-233. MR 96i:39023

[Dy] Dyson, F., Statistical theory of the energy levels of complex systems I, J. Math. Phys. 3 (1962), 140-156. MR 26:1111 
[EFK] Etingof, P.I., Frenkel, I.B. and Kirillov, A.A., Jr, Spherical functions on affine Lie groups, Duke Math. J. 80 (1995), 59-90. MR 97e:22018

[EK1] Etingof, P.I., and Kirillov, A.A.,Jr, Macdonald's polynomials and representations of quantum groups, Math. Res. Let. 1 (1994), 279-296. MR 96m:17025

[EK2] - Representation-theoretic proof of the inner product and symmetry identities for Macdonald's polynomials, Compositio Math. 102 (1996), 179-202. MR 1:394 525

[GG] Garvan, F. and Gonnet, G., Macdonald's constant term conjectures for exceptional root systems, Bull. AMS (N.S.) 24 (2) (1991), 343-347. MR 92b:33054

[Go] Good, I.J., Short proof of a conjecture by Dyson, J. Math. Phys. 11 (1970), 1884. MR 41:3290

[Gu] Gunson, J., Proof of a conjecture by Dyson in the statistical theory of energy levels, J. Math. Phys. 3 (1962), 752-753. MR 26:5908

[Ha] Habsieger, L., La q-conjecture de Macdonald-Morris pour $G_{2}$, C.R.Acad. Sci. Paris Sér. 1 Math. 303 (1986), 211-213. MR 87k:17019

[HO] Heckman, G.J., Opdam, E.M., Root systems and hypergeometric functions I, Compos. Math. 64 (1987), 329-352. MR 89b:58192a

[H1] Heckman, G.J., Root systems and hypergeometric functions II, Compos. Math. 64 (1987), 353-373. MR 89b:58192b

[H2] _ A remark on the Dunkl differential-difference operators, Harmonic analysis on reductive groups (W. Barker, P. Sally, eds.), Birkhäuser, 1991, pp. 181-191. MR 94c:20075

[H3] _ An elementary approach to the hypergeometric shift operators of Opdam, Invent.Math. 103 (1991), 341-350. MR 92i:33012

[Hu1] Humphreys, J.E., Introduction to Lie algebras and representation theory, Springer-Verlag, New York, 1972. MR 48:2197

[Hu2] , Reflection groups and Coxeter groups, Cambridge Univ. Press, Cambridge, 1990. MR 92h:20002

[J] Jack, H., A class of symmetric polynomials with a parameter, Proc. Roy. Soc. Edinburgh Sect. A 69 (1970-1971), 1-18. MR 44:6652

[K] Kadell, K., A proof of the q-Macdonald-Morris conjecture for $B C_{n}$, Mem. Amer. Math. Soc. 108 (1994). MR 94h:33013

[Ko1] Koornwinder, T.H., Special functions associated with root systems: recent progress, From Universal Morphisms to Megabytes - a Baayen Space Odyssey (K. R. Apt, A. Schrijver, \& N. M. Temme, eds.), CWI, Amsterdam, 1994, pp. 391-404.

[Ko2] _ Askey-Wilson polynomials for root systems of type BC, Hypergeometric functions on domains of positivity, Jack polynomials, and applications (D. St. Richards, ed.), Contemp. Math., vol. 138, Amer. Math. Soc., 1992, pp. 189-204. MR 94e:33039

[L] Lusztig, G., Affine Hecke algebras and their graded version, J. of the AMS 2 (3) (1989), 599-685. MR 90e: 16049

[M1] Macdonald, I.G., A new class of symmetric functions, Publ. I.R.M.A. Strasbourg, 372/S20, Actes 20 Séminaire Lotharingien (1988), 131-171.

[M2] - Orthogonal polynomials associated with root systems, preprint (1988).

[M3] , Some conjectures for root systems, SIAM J. of Math. Analysis 13 (6) (1982), 988-1007. MR 84h:17006a

[M4] The Poincaré series of a Coxeter group, Math. Annalen 199 (1972), 161-174. MR 48:433

[M5] _ Orthogonal polynomials and constant term conjectures, Lectures at Leiden University (May 1994).

[M6] - Symmetric functions and Hall polynomials, 2nd ed., Oxford Univ. Press, 1995. MR 96h:05207

[M7] _ Affine Hecke algebras and orthogonal polynomials, Séminaire Bourbaki, Vol. 199495 (797). MR 1:423 624

[Ma] Matsuo, A., Integrable connections related to zonal spherical functions, Inv. Math. 110 (1992), 95-121. MR 94g:33013

[N] Noumi, M., Macdonald's symmetric polynomials as zonal spherical functions on some quantum homogeneous spaces, Adv. in Math. 123 (1996), 16-77. MR 1:413 836

[O1] Opdam, E.M., Root systems and hypergeometric functions III, Compos. Math. 67 (1988), 21-49. MR 90k:17021 
[O2] _ Root systems and hypergeometric functions IV, Compos. Math. 67 (1988), 191209. MR 90c:58079

[O3] - Some applications of hypergeometric shift operators, Inv. Math. 98 (1989), 1-18. MR 91h:33024

[OOS] Ochiai, H., Oshima, T., and Sekiguchi, H., Commuting families of symmetric differential operators, Proc. of the Japan Acad. 70, Ser. A (2) (1994), 62-66. MR 1:272 672

[OP] Olshanetsky, M.A. and Perelomov, A.M., Quantum integrable systems related to Lie algebras, Phys. Rep. 94 (1983), 313-404. MR 84k:81007

[St] Stanley, R. Some combinatorial properties of Jack symmetric functions, Adv. Math. $\mathbf{7 7}$ (1989), 76-115. MR 90g:05020

[Su] Sutherland, B., Exact results for quantum many-body problem in one dimension, Phys. Rep. A5 (1972), 1375-1376.

[V] Verma, D-N., The role of affine Weyl groups in the representation theory of algebraic Chevalley groups and their Lie algebras, Lie groups and their representations (Proceedings of the Summer School on Group Representations), Budapest, 1971, pp. 653-705. MR 53: 13425

[W] Wilson, K., Proof of a conjecture by Dyson, J. Math. Phys. 3 (1962), 1040-1043. MR 26:2170

Department of Mathematics, Mit, Cambridge, Massachusetts 02139

E-mail address: kirillov@math.mit.edu 